\title{
The Stochastic Joint Replenishment Problem: A New Policy, Analysis, and Insights
}

\author{
Banu Yüksel Özkaya, ${ }^{1}$ Ülkü Gürler, ${ }^{2}$ Emre Berk $^{3}$ \\ ${ }^{1}$ School of Industrial and Systems Engineering, Georgia Institute of Technology, Atlanta, Georgia 30332 \\ ${ }^{2}$ Department of Industrial Engineering, Faculty of Engineering, Bilkent University, Ankara, Turkey \\ ${ }^{3}$ Department of Management, Faculty of Business Administration, Bilkent University, Ankara, Turkey
}

Received 31 December 2004; revised 25 July 2005; accepted 28 September 2005

DOI 10.1002/nav.20147

Published online 25 April 2006 in Wiley InterScience (www.interscience.wiley.com).

\begin{abstract}
In this study, we propose a new parsimonious policy for the stochastic joint replenishment problem in a singlelocation, $N$-item setting. The replenishment decisions are based on both group reorder point-group order quantity and the time since the last decision epoch. We derive the expressions for the key operating characteristics of the inventory system for both unit and compound Poisson demands. In a comprehensive numerical study, we compare the performance of the proposed policy with that of existing ones over a standard test bed. Our numerical results indicate that the proposed policy dominates the existing ones in 100 of 139 instances with comparably significant savings for unit demands. With batch demands, the savings increase as the stochasticity of demand size gets larger. We also observe that it performs well in environments with low demand diversity across items. The inventory system herein also models a two-echelon setting with a single item, multiple retailers, and cross docking at the upper echelon. (C) 2006 Wiley Periodicals, Inc. Naval Research Logistics 53: 525-546, 2006.
\end{abstract}

Keywords: multi-item inventory systems; joint replenishment problem

\section{INTRODUCTION AND LITERATURE REVIEW}

In this paper, we study the stochastic joint replenishment problem (SJRP) under a new, parsimonious policy. SJRP is the determination of replenishment and stocking decisions for $N$ different items to minimize the expected total ordering, holding, and shortage costs per unit time in the presence of random demands and ordering cost structures with firstorder interaction. The first-order-interaction structure for ordering costs is defined as the setting where there are $(i)$ a common fixed cost associated with a replenishment order regardless of its composition and (ii) an item-specific fixed cost for each item that is included in the replenishment order $[4,10]$. The ordering cost structure presents an opportunity to exploit the economies of scale in replenishment by ordering items jointly. Such joint replenishment opportunities occur when it is possible to include several different items

Correspondence to: B. Y. Özkaya (ybanu@bilkent.edu.tr); Ü. Gürler (ulku@bilkent.edu.tr); E. Berk (eberk@bilkent.edu.tr). in the same delivery order or when the items are purchased from the same supplier or they share the same transportation vehicle.

The determination of coordination and control mechanisms for multi-item inventory systems is a real problem faced by retailers and is an integral part of supply chain management in general. Moreover, it is becoming an increasingly important problem due to the recent trend among manufacturers and retailers to reduce their supplier bases [14]. It is estimated that major Original Equipment Manufacturers (OEMs) have reduced the number of their suppliers by a factor of 4 since the mid-1990s. A best practice study reports that world-class companies operate with $97 \%$ fewer suppliers for A-category items, when compared with the average (The Hackett Group, www.thehackettgroup.com). Another survey reveals that $80 \%$ of the firms directly considered the potential cost savings due to the reduction of transaction costs among multiple suppliers [6]. In their recent works, Erhun and Tayur [8] and Cachon [5] also report particular instances of considerable cost savings achieved by exploiting the economies of scale due to joint replenishment opportunities. 
Despite its practical importance, solution of the stochastic joint replenishment problem is notoriously difficult. To our knowledge, Ignall [15] authored the only study that attempts to find the structure of the optimal joint replenishment policy with stochastic demand. The optimal policy may have a very complex structure even for two items with zero lead time, due to the dependence between the order quantity of an item and the inventory level of the other at an ordering instance. Based on this finding, one may conjecture that the optimal policy for $N$ items would involve control surfaces defined by the inventory levels of other items considered in the replenishment. Even if the exact structure is found, it would be too complex to compute and implement it in practice. Hence, most of the existing approaches to the problem have been confined to the evaluation of some intuitive policy classes that are relatively easy to compute and implement. It mainly follows from their heuristic nature that the policies in the literature do not dominate each other uniformly over the entire parameter space, as demonstrated by previous works and the numerical study herein.

The stochastic joint replenishment problem differs from its deterministic counterpart (JRP) greatly in terms of modeling methodologies and the employed policy structures arising from the deterministic nature of demand. Therefore, the vast body of research on JRP falls outside the scope of this study. We refer the reader to Aksoy and Erenguc [1] and Goyal and Satir [12] for extensive reviews of the works in deterministic demand environments. The literature on the stochastic joint replenishment problem can be classified into two major streams based on the type of policy class under consideration. In our review, we follow this classification.

\subsection{Can-Order Policies}

This stream of research has begun with the earliest work on joint replenishment with stochastic demand by Balintfy [4], who introduced the continuous review (s, c, S) joint ordering policy-also called the can-order policy. The policy operates as follows. When the inventory position of an item $i$ crosses $s_{i}$, a replenishment order is triggered to raise its inventory position to $S_{i}$. At the same time, any other item $j$ with an inventory position at or below its can-order point, $c_{j}\left(s_{j}<c_{j}<S_{j}\right)$, is also included in the replenishment, raising its inventory position to $S_{j}$. Despite its benign structure, the analytical treatment of the system under this policy is extremely difficult even in the presence of unit Poisson demands. Balintfy [4] only provides an initial insight into the problem with a queuing-based approach. A special case with $\mathbf{c}=\mathbf{S}-\mathbf{1}$ and $\mathbf{s}=\mathbf{0}$ in a two-item inventory system facing identical unit Poisson demands with zero lead time has been analyzed by Silver [25]. Under the assumption that shortages are not allowed and with the objective of minimizing ordering and holding costs per unit time, Silver [25] proves that the can-order policy is always better than independent control if the cost of placing an order for two items is equal to that for a single item; and, otherwise, there exists a critical value of the joint ordering cost only below which it is preferable to use joint replenishment. An exact analysis has been possible for this special case because the inventory levels of both items provide regeneration points at the order instances and, hence, the renewal reward theorem is applicable. However, the same approach cannot be used for the general case. Therefore, different approximate models and solution methods have been proposed in the literature.

A common approximation technique proposed by Silver [27] is to decompose the $N$-item problem with unit Poisson demands into $N$ single-item problems facing unit Poisson demands and Poisson special replenishment opportunities. The resulting single-item problem has been analyzed by Silver [26] and solved optimally by Zheng [34]. The same decomposition technique has later been extended to compound Poisson demand by Thompson and Silver [31] and Silver [28]. Using a similar decomposition approach, Federgruen, Greoenvelt, and Tijms [9] propose a semi-Markov decision model and use a policy-iteration algorithm to solve for the optimal values of the control policy parameters. We denote this policy by $(\mathbf{s}, \mathbf{c}, \mathbf{S})_{F}$. Van Eijs [32] and Schultz and Johansen [24] have illustrated that the decomposition method assuming a Poisson arrival process for the special replenishment opportunities can lead to poor performance of the can-order policies. Instead, they propose using Erlang distributions in the decomposition. The optimal values of the policy parameters are obtained through policy iteration and simulation-based updating of the stochastic process governing the opportunities. Melchiors [18] proposed to use a new compensation approach and was able to improve the previous approximations of the continuous can-order policies for unit Poisson demands. We denote this policy by (s, c, $\mathbf{S})_{M}$. However, the approach and the approximations used require extensive iterative computations and may result in significant deviations from simulated costs in some cases. Recently, Johansen and Melchiors [16] proposed a periodic review version of the can-order policy, which performs well when there is high demand variation across the items.

As the above summary indicates, almost all of the works on the can-order policy have focused on alleviating the inherent modeling complexities arising from the nature of the policy class. Another major difficulty with the can-order policy is the size of the optimization problem. For an $N$-item setting, the continuous review $(\mathbf{s}, \mathbf{c}, \mathbf{S})$ policy employs $3 N$ control policy parameters, whereas the periodic review counterpart has $3 N+1$ policy parameters. For realistic operating environments, this implies extensive numerical optimization effort. Coupled with the iterative nature of the decomposition techniques developed in the literature, the can-order policy appears to be a prohibitively tedious con- 
trol policy class. Therefore, a number of researchers have proposed control policies that are more parsimonious (i.e., with fewer control policy parameters) and/or easier to model and optimize. We discuss such policies next.

\subsection{Other Policies}

The continuous review $(Q, \mathbf{S})$ policy was first proposed by Renberg and Planche [22], and subsequently studied by Pantumsinchai [21] with Poisson demand. Under the $(Q, \mathbf{S})$ policy, when the aggregate consumption since the previous order reaches $Q$, all items are raised up to the vector of order-up-to levels, $\mathbf{S}$. The policy employs $N+1$ policy parameters in an $N$-item setting. An exact analysis is presented in Pantumsinchai [21] and the numerical findings indicate that the performance of $(Q, \mathbf{S})$ policy vis a vis the can-order policy is remarkable for high ordering cost, small number of items, and low shortage costs, whereas the latter performs better only with small ordering costs. Atkins and Iyogun [3] propose two base-stock periodic review policies for unit Poisson demands, developed on the basis of a lower bound on the cost rate established previously by the authors [2]. The first policy, $P$, imposes the same review period length $T$ for all items, and the inventory levels of all items are raised to their order-up-to levels defined by $\mathbf{S}$. The policy employs $N+1$ policy parameters. The second policy $M P$ is a modified periodic policy that utilizes itemspecific review period lengths based on the afore-mentioned lower bound; it uses $2 \mathrm{~N}$ policy parameters. Their numerical study indicates that the proposed policies dominate the (s, c, S) policy except when the ordering costs are small. As reported by Pantumsinchai [21], the performance of the $M P$ policy is comparable to that of the $(Q, \mathbf{S})$ policy.

Viswanathan [33] recommends a new policy class. Under the proposed policy, $P(\mathbf{s}, \mathbf{S})$, one uses an independent, periodic review $(s, S)$ policy for each item with a common review interval, $T$. This policy employs $2 N+1$ policy parameters for an $N$-item setting. An approximate solution is provided under the assumption that an order is placed at each review epoch. An extensive comparison of the $P(\mathbf{s}, \mathbf{S})$ policy is made with the $M P,(Q, \mathbf{S}),(\mathbf{s}, \mathbf{c}, \mathbf{S})$ policies. $P(\mathbf{s}$, $\mathbf{S})$ dominates the other policies especially when the holding costs are high compared to the backorder costs.

In a very recent study, Nielsen and Larsen [20] proposed the $Q(\mathbf{s}, \mathbf{S})$ policy in which inventories are reviewed only when $Q$ total demands accumulate since the last review instance. At the review instance, any item $j$, the inventory position of which is less than or equal to its reorder level $s_{j}$, is ordered up to $S_{j}$. This policy employs $2 N+1$ policy parameters for an $N$-item setting. In the operating environments with identical demand and cost structures for the items, the policy reduces to the $(Q, \mathbf{S})$ policy. Over a small test bed, the policy was superior to the previously proposed policies. However, as will be demonstrated in our numerical study below, the new policy proposed herein dominates $Q(\mathbf{s}, \mathbf{S})$ in the vast majority of cases considered in a standard test bed.

As the above discussion of the existing policies illustrates, the stochastic joint replenishment problem is an open research area for the development of more efficient computational methods and control policies. For the latter, we believe that the parsimony of the policies and the robustness of their performance are the main criteria to judge by.

In this study, we propose a new class of control policy for SJRP that makes use of the advantages of both continuous and periodic review policies in a parsimonious manner. The $(Q, \mathbf{S}, T)$ policy, proposed herein, bases the joint ordering decisions on the accumulation of $Q$ demands or the time elapsed $(T)$ since the last decision epoch, whichever occurs first. As such, it is a hybrid extension of the $(Q, \mathbf{S})$ and $P$ policies and uses only $N+2$ control policy parameters for an $\mathrm{N}$-item setting. Despite the low dimensionality of the proposed policy, our numerical study indicates that it performs well in comparison with the existing policies. Across all 139 instances in a standard (Atkins-Iyogun and Viswnanathan) test bed, we see that, among all the policies considered, the proposed policy gives the least cost rate in 100 instances. It achieved an overall average improvement of $1.14 \%$ with a maximum of $3.55 \%$ over the next best policy. As discussed in our numerical section, this constitutes comparably considerable savings in operating environments with multiple items and relatively low profit margins. Moreover, the proposed policy attains such performance levels with parsimony $(N+2$ policy parameters for $N$ items). This parsimony reduces the computational effort in optimization enormously and eases implementation in practice greatly. Viewing the comparison in this broader perspective, we believe that the proposed policy and the model developed herein provide significant improvements over the existing models in terms of cost savings, optimization effort, and ease of implementation. Although we motivate our model in a single-location, multi-item setting, it can also be used in a two-echelon, single-item, multi-retailer setting with cross docking at the upper echelon. Given the increasing use of cross docking in retail industry (e.g., Wal-Mart Stores), the model and findings herein have important implications for supply chain design and management as well.

The rest of the paper is organized as follows: Section 2 states the main assumptions of the model and introduces the new joint ordering policy under unit Poisson demands. In Section 3, we develop the expressions for the key operating characteristics of the inventory system. In Section 4, we generalize the proposed policy to a case with compound Poisson demand. In Section 5, numerical results are presented on the performance of the proposed policy in comparison with the previously proposed joint replenishment 
policies and on the sensitivity of the policy parameters. We conclude with some remarks in Section 6.

\section{THE MODEL}

We consider a continuous review, multi-item inventory system with $N \geq 2$ items facing unit external demands generated by independent and stationary Poisson processes with rate $\lambda_{i}(i=1,2, \ldots, N)$. (We relax this assumption in Section 4.) All unmet demands are assumed to be backordered. Items are supplied from an ample supplier and delivery lead times are constants given by $L_{i}$ for item $i$. (We do not restrict our analysis to identical lead times. Thus, our model allows us to consider joint replenishment decisions through routing of vehicles among various locations in a two-echelon, single-item, multi-location setting with cross docking at the depot level as well.) The system is continuously reviewed and, hence, the records for the last replenishment epoch, as well as the time elapsed since then and the total demand arrived to the system after the last order are available. The fixed ordering costs in the system have two components: a common ordering cost, $K$, which is charged every time a replenishment order is placed, and a fixed item specific ordering cost $k_{i}$, for item $i$ that is added if item $i$ is included in the order. Holding cost is charged at $h_{i}$ per unit of item $i$ held in stock per unit time. Two types of shortage costs are incurred: a time weighted shortage cost at $\rho_{i}$ per unit backordered of item $i$ per unit time and a fixed penalty cost of $\pi_{i}$ for every unit of item $i$ unable to be satisfied immediately on demand arrival. We ignore the unit purchasing costs since all demand is eventually satisfied.

Under the assumed cost structure, the objective is to minimize the expected total cost per unit time. We propose below a joint replenishment policy that unifies the time and inventory position considerations for the placement of orders. Note that inventory position at any point in time is defined as on-hand inventory plus on order minus backorders. The policy is formally stated as below:

Policy: Monitor all inventory positions continuously, and raise the inventory positions of the items up to $\mathbf{S}=\left(S_{1}\right.$, $S_{2}, \ldots, S_{N}$ ) whenever a total of $Q$ demands accumulate for the items or $T$ time units have elapsed, whichever occurs first.

We shall refer to the proposed policy above as the $(Q$, $\mathbf{S}, T)$ policy, where $\mathbf{S}$ is the vector denoting the maximum inventory positions of the items, and $T$ and $Q$ correspond, respectively, to the time and inventory triggers. In the sequel, we use the term "decision epoch" to refer to an instance at which either a replenishment order is placed or merely an inventory review is made without any order placement. Suppose, for example, that a total of $Q$ de- mands have arrived before $T$ time units have elapsed since the last decision epoch; then an order is placed at the instance of the $Q$ th demand arrival, which constitutes a decision epoch. Suppose alternatively that $T$ time units have elapsed before a total of $Q$ demands have arrived. At this instance, an inventory review may or may not result in an order placement. If at least one demand has arrived in $T$ units of time, reordering will occur and the placement of an order constitutes the decision epoch. However, if no demand has arrived within the $T$ units of time, then the decision is not to order anything, and the decision epoch coincides with merely an inventory position review instance. Due to the Poisson demand process, we immediately see that decision epochs constitute regenerative instances for the system.

The $(Q, \mathbf{S}, T)$ policy is a hybrid of the continuous review $(Q, \mathbf{S})$ policy, first proposed by Renberg and Planche [22], and the periodic review $(\mathbf{R}, T)$ policy of Atkins and Iyogun [3]. Thus, it attempts to exploit the benefits of two separate policies. As expected, it reduces to these two policies in the limit: as $T \rightarrow \infty$, we obtain the $(Q, \mathbf{S})$ policy; and, as $Q \rightarrow$ $\infty$, we obtain the $(\mathbf{R}, T)$ policy. The replenishment quantity under the $(Q, \mathbf{S}, T)$ policy is a random variable; it may be as small as 1 unit and cannot exceed $Q$ units. This is in contrast with the $(Q, \mathbf{S})$ policy, which imposes a constant reorder size. Hence, the $(Q, \mathbf{S}, T)$ policy may not fully exploit the economies of scale in joint ordering in every order instance in comparison with the $(Q, \mathbf{S})$ policy. We have observed this disadvantage in some cases in our numerical results (see Section 3.2). However, the cause of this diseconomy, namely, the introduction of the time trigger, $T$, helps in another way and compensates for this inefficiency. Under the $(Q, \mathbf{S})$ policy, the inter-order times are random. To be specific, they have Erlang_ $Q$ distribution, which may have quite long tails. The introduction of $T$ cuts such long tails, as it imposes an upper bound on the time between two consecutive decision epochs (and, thereby, reorder times). Furthermore, the presence of a time-based reorder trigger provides the opportunity of pro-active reordering in the presence of non-Markovian total demand process. We know from Katircioglu [17] that a time-based reorder trigger is optimal for single-location models with non-Markovian demands (see also [19]). Similarly, Tekin, Gürler, and Berk [30] show that such a policy class performs better for a special perishable inventory system as well. Hence, we would expect the introduction of $T$ to improve the $(Q, \mathbf{S})$ policy. Our numerical experiments have confirmed this, as will be discussed in more detail in the numerical results section.

Next, we present some preliminary results needed to derive the operating characteristics of the system. 

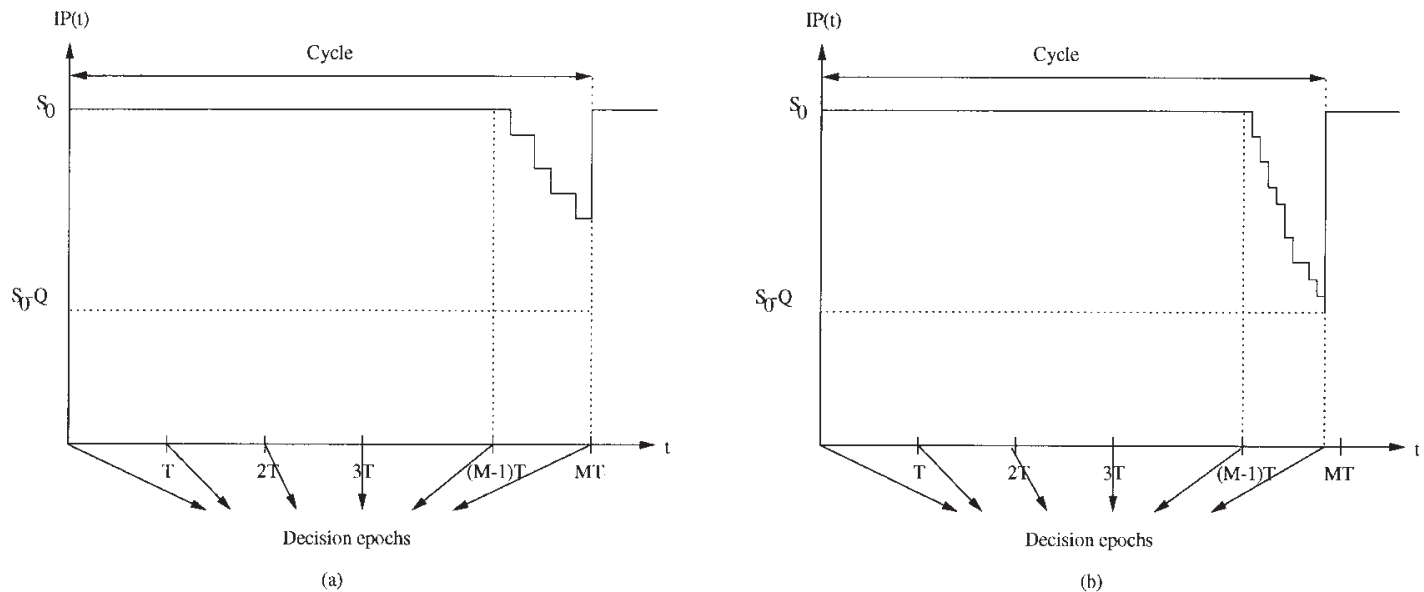

(b)

Figure 1. Realizations for a cycle.

\subsection{Preliminary Analysis}

In this subsection, we obtain two entities: the joint distribution of the order size and the inter-order time; and the steady-state distribution of the individual inventory positions of the items.

First, we introduce some notation. Let $r_{i}$ be the probability that the demand is for item $i$, given that a demand arrival has occurred. Since the demand process is Poisson, $r_{i}=$ $\lambda_{i} / \lambda_{0}$, where $\lambda_{0}=\sum_{j=1}^{N} \lambda_{j}$ is the system demand rate. Let $X_{n}, n=1,2, \ldots$ denote the random variable representing the arrival time of the $n$th system demand after the last decision epoch, which could be either a demand instance or a time trigger. Since inter-arrival times of the demands are exponential, the time until next demand (forward recurrence time for the demand process since the last decision epoch) is also exponential and therefore $X_{n}$ has an Erlang_ $n$ distribution with scale parameter $\lambda_{0}$. Also, let $f(x, k, \lambda)$ and $F(x, k, \lambda)$ be the probability density and distribution function of an Erlang random variable with shape and scale parameters $k$ and $\lambda$, respectively. For any distribution function $F$, we use $\bar{F}=1-F$.

Under the $(Q, \mathbf{S}, T)$ policy, we define a cycle as the time between two consecutive order placement decisions. A cycle starts every time a positive replenishment order is given (raising the inventory positions to $\mathbf{S}$ ). Under the proposed policy, there may be multiple decision epochs, separated by intervals of length $T$ within a cycle. We denote the total number of such decision epochs by $M$, which is a geometric random variable. We present two realizations of the evolution of a cycle in Figure 1.

Figure 1a refers to a realization where, in the first $(M-$ $1) \geq 0$ intervals of length $T$ since the last order placement decision, no demand has arrived and in the next interval of length $T$, less than $Q$ but more than 1 demands have arrived to the system, triggering a reorder decision based on the time threshold. Hence, the length of the cycle is $M T$. Figure $1 \mathrm{~b}$ refers to a realization where, in the first $(M-1)$ intervals of length $T$ since the last order placement decision, no demand has arrived as in Figure 1a, but before $T$ more time units elapse, $Q$ demands arrive, triggering a replenishment. Hence, the length of the cycle is random with a value between $(M-1) T$ and $M T$. As mentioned above, $M$ is a random variable that is geometrically distributed, with parameter $\phi_{0}=p_{0}\left(0, \lambda_{0}, T\right)$, where $p_{0}(x, \lambda)$ denotes the probability mass function of a Poisson random variable at $x$, with rate $\lambda$.

For clarity and later use, we make the following definitions. Let $I P_{i}(t)$ denote the inventory position of item $i$ and $I P(t)$ denote the total inventory position of the system at time $t$. Then, $I P(t)=\sum_{i=1}^{N} I P_{i}(t) \leq \sum_{i=1}^{N} S_{i}=S_{0}$. Also let $N I_{i}(t)$ denote the net inventory level of item $i$ at time $t$. In order to illustrate the behavior of the inventory system under the proposed policy, we depict a particular realization in Figure 2. Figures $2 \mathrm{a}$ and $\mathrm{b}$ show the inventory positions and net inventory of item 1 and item 2, respectively. Figure $2 \mathrm{c}$ displays the corresponding total inventory position. In the following, we briefly narrate the time sequence of the events and the decisions taken. In this illustration, we have $S_{1}=5, S_{2}=3, Q=3$, and some $T>0$ as the policy parameters; initially both items are at their maximum stocking levels. For generality, we assume that lead times for individual items are different. That is, an order consisting of units for both items will be received at different times by the two items. For illustration, we assume $L_{1}>L_{2}>0$. At time $t=t_{1}$, a demand arrives for item 1. At $t=t_{2}$, a demand arrives for item 2. At time $t=t_{3}(<T)$, another demand arrives for item 1 . At this instance, the number of demands accumulated in the system reaches $Q=3$. This triggers an order placement at $t=t_{3}$, which brings the inventory position of item 1 to $S_{1}$ and of item 2 to $S_{2}$. This 

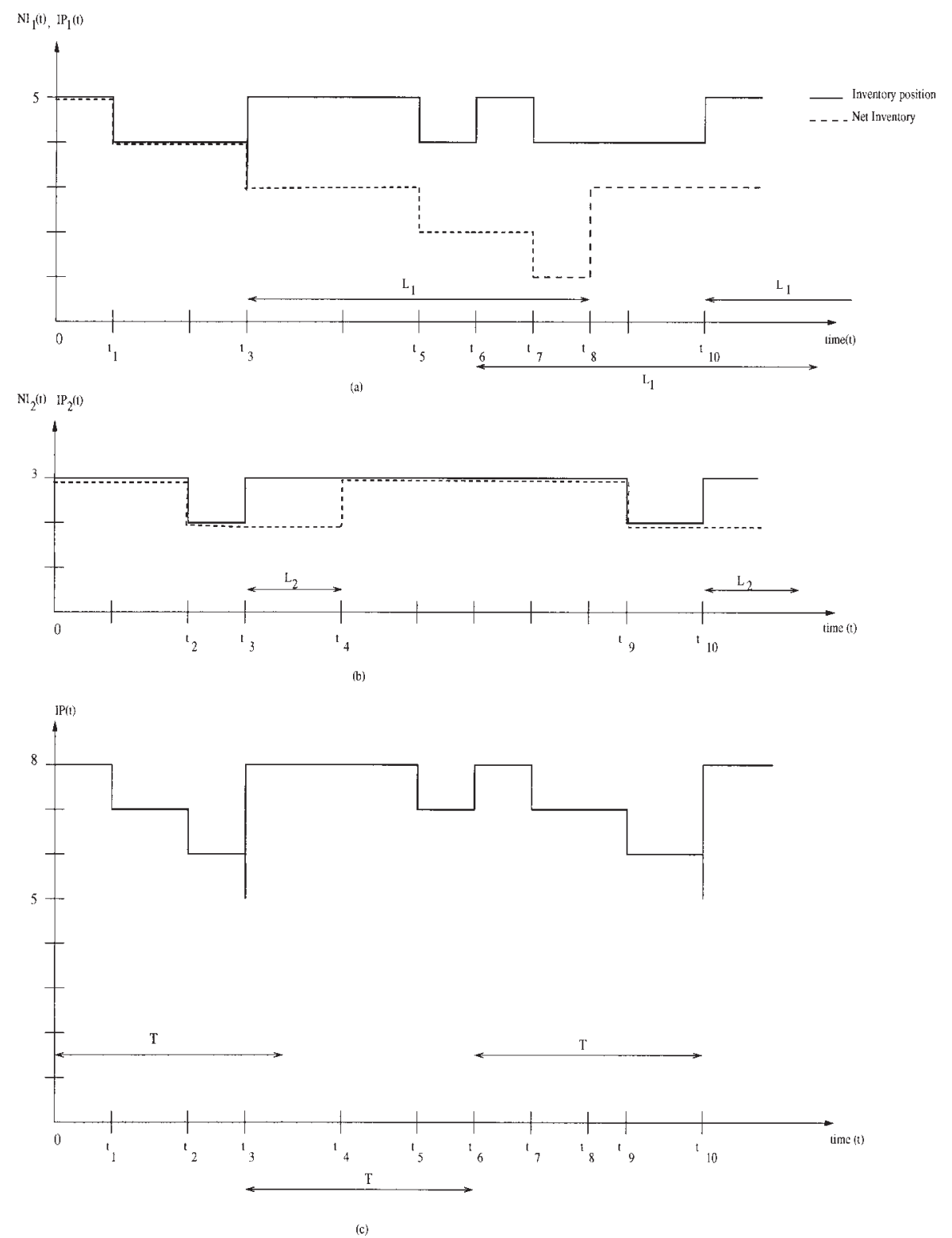

Figure 2. Evolution of ordering process.

order consists of three units, two of which are for item 1 and the remaining one unit is for item 2. At this point, there is one outstanding order in the system and both items are awaiting some delivery. At time $t_{4}=t_{3}+L_{2}$, the unit for item 2 in the order placed at $t_{3}$ arrives, raising the net inventory of item 2 to three. At time $t_{5}$, a demand arrives for item 1 and drops its inventory position to four and its net inventory to two (since item 1 is still awaiting its delivery). At time $t_{6}=t_{3}+T$, a total of $T$ time units have elapsed since the last order was placed; therefore, an order is placed as triggered by the policy. The order size is 1 and only item
1 is included in this order since no demand has arrived for item 2 between $t=t_{3}$ and $t=t_{6}$. At time $t_{7}$, another demand arrives for item 1 decreasing its inventory position to four and its net inventory to one. Note that, between $t_{6}$ and $t_{8}=t_{3}+L_{1}$, there are two outstanding orders for item 1 whereas there is no outstanding order for item 2 . At time $t=t_{8}$, the units in the order given at time $t_{3}$ are received by item 1 and its net inventory is raised to three. A demand for item 2 arrives at time $t=t_{9}$ dropping both the inventory position and net inventory to two. At time $t_{10}=t_{6}+T$, another order is placed; its order size is two, with one unit 
for each item. At $t_{10}$, there are two outstanding orders for item 1 and one outstanding order for item 2. The process goes on further.

Let $Y$ and $Q_{0}$ denote random variables corresponding to the cycle length (i.e., the inter-order time) and the order size, respectively. For convenience, we shall use the term "joint density" for joint density/probability mass function of random vectors when some components are discrete and others are continuous random variables. Let $f_{Y, Q_{0}}(y$, $q$ ) denote the joint probability density function of $Y$ and $Q_{0}$. Then, we have the following (as proved in the Appendix).

\section{Lemma 2.1:}

$$
f_{Y, Q_{0}}(y, q)=\left\{\begin{array}{l}
\phi_{0}^{m-1} p_{0}\left(q, \lambda_{0} T\right) \\
\quad \text { if } y=m T, m \geq 1,0<q<Q \\
\phi_{0}^{m-1} f\left(y-(m-1) T, Q, \lambda_{0}\right) \\
\quad \text { if }(m-1) T<y<m T, m \geq 1, q=Q
\end{array}\right.
$$

Using the above lemma, we can find the marginals, which will be of use in the sequel.

Corollary 2.1:

(a) The probability mass function $P_{Q_{0}}(q)=P\left(Q_{0}=\right.$ $q$ ) of $Q_{0}$ is given by

$P_{Q_{0}}(q)$

$$
= \begin{cases}p_{0}\left(q, \lambda_{0} T\right) /\left(1-\phi_{0}\right) & \text { if } 0<q<Q \\ \bar{P}_{0}\left(Q-1, \lambda_{0} T\right) /\left(1-\phi_{0}\right) & \text { if } q=Q .\end{cases}
$$

(b) The p.d.f., $f_{Y}(y)$, of $Y$ is given by

$$
f_{Y}(y)=\left\{\begin{array}{c}
\phi_{0}^{m-1}\left[P_{0}\left(Q-1, \lambda_{0} T\right)-\phi_{0}\right] \\
\text { if } m \geq 1, y=m T \\
\phi_{0}^{m-1} f\left(y-(m-1) T, Q, \lambda_{0}\right) \\
\quad \text { if } m \geq 1,(m-1) T<y<m T
\end{array}\right.
$$

where $P_{0}(x, \lambda)$ denotes the Poisson cumulative distribution function with rate $\lambda$.

Next, we will obtain the steady-state inventory positions of the items.

As already mentioned, each decision epoch is a regeneration point for the system, since the inventory positions of all the items are at their base-stock levels at these instances under the $(Q, \mathbf{S}, T)$ policy. Hence, we know that the steady-state distributions of the inventory positions of items exist (see [29]).
For $t>0$ and $1 \leq i \leq N$, define the three-dimensional stochastic process, $\xi_{i}(t)=\left\{N_{i}(t), N_{0}(t), Z(t)\right\}$, where $Z(t)$ denotes the time between $t$ and the last decision epoch and $N_{i}(t)$ and $N_{0}(t)$ denote, respectively, the number of demands for item $i$ and for all other items that have arrived over $Z(t)$ time units. A particular state that $\xi_{i}(t)$ visits at time $t$ will be denoted by $\left\{n_{i}, n_{0}, z\right\}$. Then, $g_{i}\left(t, n_{i}, n_{0}, z\right)$ denotes the probability density function of $\xi_{i}(t)$. Assuming that a steady-state density exists, we have the following result:

Proposition 2.1: The steady state p.d.f., denoted by $g_{i}\left(n_{i}\right.$, $\left.n_{0}, z\right)$ is given by the expression

$$
g_{i}\left(n_{i}, n_{0}, z\right)=C_{0} p_{0}\left(n_{i}, \lambda_{i} z\right) p_{0}\left(n_{0},\left(\lambda_{0}-\lambda_{i}\right) z\right)
$$

for $0<z<T$ and $0 \leq n_{0}+n_{i} \leq Q-1, n_{0} \geq 0, n_{i} \geq$ 0 , where $C_{0}$ is the normalizing constant and given by

$$
C_{0}=\left[\int_{t=0}^{T} P_{0}\left(Q-1, \lambda_{0} t\right) d t\right]^{-1}
$$

Proof rests on the development of the partial differential equations describing the dynamics of the stochastic process, $\xi_{i}(t)$, via supplementary variables and is provided in the Appendix. (See [7] and [23] for details of the technique.)

Due to the nature of the control policy that ensures constant inventory positions at decision epochs, there is a one-to-one correspondence between observed demands and inventory positions of items. If $n_{i}$ demands have arrived for item $i$ after the last decision epoch, the inventory position of item $i$ is $S_{i}-n_{i}$. Hence, from Proposition 2.1 , we can immediately obtain the steady-state distribution of the inventory position of item $i$.

Proposition 2.2: Let $\varphi_{i}(x)$ denote the steady-state probability that the inventory position of item $i$ is $x$. Then,

$$
\begin{array}{r}
\varphi_{i}\left(S_{i}-n_{i}\right)=\frac{C_{0}}{\lambda_{0}} \sum_{n_{0}=0}^{Q-1-n_{i}}\left(\begin{array}{c}
n_{0}+n_{i} \\
n_{i}
\end{array}\right) r_{i}^{n_{i}}\left(1-r_{i}\right)^{n_{0}} F\left(T, n_{0}\right. \\
\left.+n_{i}+1, \lambda_{0}\right)
\end{array}
$$

for $0 \leq n_{i} \leq Q-1$.

Proof: Using Proposition 2.1, we have 


$$
\begin{aligned}
\varphi_{i}\left(S_{i}-n_{i}\right)=\sum_{n_{0}=0}^{Q-1-n_{i}} \int_{z=0}^{T} g_{i}\left(n_{i}, n_{0}, z\right) d z=C_{0} \sum_{n_{0}=0}^{Q-1-n_{i}} \int_{z=0}^{T} p_{0}\left(n_{i}, \lambda_{i} z\right) p_{0}\left(n_{0},\left(\lambda_{0}-\lambda_{i}\right) z\right) d z \\
=C_{0} \sum_{n_{0}=0}^{Q-1-n_{i}} \int_{z=0}^{T} \frac{e^{-\lambda_{i} z}\left(\lambda_{i} z\right)^{n_{i}}}{n_{i} !} \frac{e^{-\left(\lambda_{0}-\lambda_{i}\right) z}\left(\left(\lambda_{0}-\lambda_{i}\right) z\right)^{n_{0}}}{n_{0} !} d z=C_{0} \sum_{n_{0}=0}^{Q-1-n_{i}} \frac{\lambda_{i}^{n_{i}}\left(\lambda_{0}-\lambda_{i}\right)^{n_{0}}}{\lambda_{0}^{n_{i}+n_{0}+1}} \frac{\left(n_{0}+n_{i}\right) !}{n_{0} ! n_{i} !} \int_{z=0}^{T} \\
\lambda_{0} \frac{e^{-\lambda_{0} z}\left(\lambda_{0} z\right)^{n_{0}+n_{i}}}{\left(n_{0}+n_{i}\right) !} d z=\frac{C_{0}}{\lambda_{0}} \sum_{n_{0}=0}^{Q-1-n_{i}}\left(\begin{array}{c}
n_{0}+n_{i} \\
n_{i}
\end{array}\right) p_{i}^{n_{i}}\left(1-p_{i}\right)^{n_{0}} F\left(T, n_{0}+n_{i}+1, \lambda_{0}\right) .
\end{aligned}
$$

Now, we are ready to formulate the operating characteristics of the inventory system.

\section{OPERATING CHARACTERISTICS}

In this section, we derive the expressions for the expected cycle length, the order placement rate, and the expected values of the steady-state on-hand inventory and backorder levels. These expressions are then used to construct the expected cost rate function.

We begin with expected cycle length, $E[Y]$. As detailed in the Appendix, we have

$$
E[Y]=\frac{T P_{0}\left(Q-1, \lambda_{0} T\right)}{1-\phi_{0}}+\frac{Q \bar{P}_{0}\left(Q, \lambda_{0} T\right)}{\lambda_{0}\left(1-\phi_{0}\right)} .
$$

In each cycle, the common ordering cost is incurred once. Hence, the common ordering cost rate is simply $K / E[Y]$. In each replenishment, item-specific ordering costs are also incurred. To obtain the item-specific ordering cost rate, one must find the items that are included in any given order. The probability that item $i$ is included in an order of size $q(1<$ $q<Q)$ is $1-\left(1-r_{i}\right)^{q}$, where $r_{i}=\lambda_{i} / \lambda_{0}$ as defined before. Letting $\theta_{i}$ denote the probability that item $i$ is included in a replenishment order, we have

$$
\theta_{i}=\sum_{q=1}^{Q} P_{Q_{0}}(q)\left[1-\left(1-r_{i}\right)^{q}\right]
$$

where $P_{Q_{0}}(q)$ is given in Corollary 2.1.

To compute the expected on-hand inventory level and the expected number of backorders at any time, we employ the standard argument of Hadley and Whitin [13] as follows: Consider the system at time instances $t$ and $t+L_{i}$, where $L_{i}$ is the constant replenishment leadtime of item $i$. Note that all outstanding orders at time $t$ and no orders placed afterward will have arrived by time $t+L_{i}$. Hence, we can find the steady-state inventory levels at time $t+L_{i}$ by

Naval Research Logistics DOI 10.1002/nav conditioning on the steady-state distribution of the inventory position at time $t$.

At steady state, we have the probability mass function of on-hand inventory level $\mathrm{OH}_{i}$ and backorder level, $B O_{i}$ as follows:

$$
\begin{aligned}
P\left(O H_{i}=y_{i}\right)=\sum_{n_{i}=S_{i}-Q+1}^{\min \left(S_{i}, y_{i}\right)} \varphi_{i}\left(n_{i}\right) p_{0}\left(n_{i}-y_{i}, \lambda_{i} L_{i}\right) & 0 \leq y_{i} \\
& \leq S_{i}
\end{aligned}
$$

$$
P\left(B O_{i}=y_{i}\right)=\sum_{n_{i}=S_{i}-Q+1}^{S_{i}} \varphi_{i}\left(n_{i}\right) p_{0}\left(n_{i}+y_{i}, \lambda_{i} L_{i}\right) \quad y_{i} \geq 0 .
$$

The above follows from the independence of the leadtime demand and the inventory position, since the demand process is Poisson.

Hence, at steady state, we have $E\left[O H_{i}\right]$ and $E\left[B O_{i}\right]$ as follows:

$$
\begin{aligned}
& E\left[O H_{i}\right]=\sum_{y_{i}=1}^{S_{i}} y_{i} P\left(O H_{i}=y_{i}\right) \\
& E\left[B O_{i}\right]=\sum_{y_{i}=1}^{\infty} y_{i} P\left(B 0_{i}=y_{i}\right) .
\end{aligned}
$$

The steady-state probability that there is no stock on hand of item $i, \psi_{i}$ is given as

$$
\psi_{i}=1-\sum_{y_{i}=1}^{S_{i}} P\left(O H_{i}=y_{i}\right)
$$

We also define the system fill rate, $\phi_{0}$, as 


$$
\phi_{0}=1-\sum_{i=1}^{N} r_{i} \psi_{i}
$$

We can now construct the expected cost rate $A C(Q, \mathbf{S}, T)$ for the whole system using Eqs. (2)-(8).

$$
\begin{gathered}
A C(Q, \mathbf{S}, T)=\frac{K+\sum_{i=1}^{N} k_{i} \theta_{i}}{E[Y]}+\sum_{i=1}^{N} h_{i} E\left[O H_{i}\right] \\
+\sum_{i=1}^{N} \rho_{i} E\left[B O_{i}\right]+\sum_{i=1}^{N} \pi_{i} \lambda_{i} \psi_{i}
\end{gathered}
$$

Then, the optimization problem is minimization of $A C(Q$, $\mathbf{S}, T)$ with respect to $Q, \mathbf{S}$, and $T$.

Although an explicit expression is provided in Proposition 2.1 for the steady-state distribution of inventory positions, the complicated nature of the expressions of the operating characteristics does not allow for an analytical investigation of the unimodality or the convexity of the objective function. We comment on our numerical observations in this regard in the next section.

\section{EXTENSION TO COMPOUND POISSON DEMAND}

Although unit Poisson demand assumption is commonly made in inventory models, the Poisson distribution may exhibit a poor fit to data in certain environments since it may not capture the variability of demands sufficiently. In this section, we therefore extend our results to a general setting where items face batch demands that arrive according to a Poisson process but with a random batch size that is independent of the arrivals. Specifically, we assume that customers who demand item $i$ arrive according to a Poisson process with rate $\lambda_{i}$ and demand $x$ units of item $i$ with probability $v_{i}(x)$ for $i=1,2, \ldots, N$ and $x=1,2, \ldots$. Let $v_{i}^{(k)}(x)$ for $k=1,2, \ldots$ and $x=1,2, \ldots$ denote the probability that $x$ units of item $i$ have been demanded by $k$ customers who arrived for item $i$. Incidentally, $v_{i}^{(k)}(x)$ is the $k$ th convolution of the demand size distribution $v_{i}(x)$. We retain all of the other assumptions and the corresponding notation introduced in Sections 2 and 3. Additionally, we assume that if the on-hand inventory is not sufficient to satisfy fully an arriving customer's demand, the demand is partially filled with the available stock and the rest is backordered. We propose in the following the generalized $(Q, \mathbf{S}$, T) policy.

Policy: Monitor all inventory positions continuously, and raise the inventory positions of the items up to $\mathbf{S}=\left(S_{1}\right.$,
$S_{2}, \ldots, S_{N}$ ) whenever the total inventory position crosses $S_{0}-Q$ or $T$ time units have elapsed since the last decision epoch, whichever occurs first, where $S_{0}=\sum_{i=1}^{N} S_{i}$.

There are two fundamental differences between the unit and compound Poisson demand cases: $(i)$ the order size may now exceed $Q$ units since the total inventory position is allowed to cross $S_{0}-Q$, and (ii) the number of units demanded in a replenishment cycle may not be equal to the number of customer arrivals since each customer may demand more than one unit of an item. The derivation of the expressions for the operating characteristics for the compound Poisson case is based on the methodology used for the unit demands but is modified slightly to account for the mentioned differences as explained below.

Let $\mathcal{N}$ denote the set of all the items comprising the inventory system and $\Omega$ denote a subset. Also let $w_{\Omega}(q, k)$ be the probability that $k$ customers demand a total of $q$ units for the items in the set $\Omega$. Then, for $q \geq k \geq 1, \Omega=\{i\}$, $i=1,2, \ldots, N, w_{\Omega}(q, k)=v_{\Omega}^{(k)}(q)$. For $q \geq k \geq 1$, $\Omega=\mathcal{N}$, we have

$$
\begin{aligned}
& w_{\Omega}(q, k)=\sum_{\left\{\begin{array}{c}
\sum_{i=1}^{N} x_{i}=k \\
\sum_{i=1}^{N} q_{i}=q
\end{array}\right\}} \frac{k !}{x_{1} ! x_{2} ! \ldots x_{N} !} r_{1}^{x_{1}} r_{2}^{x_{2}} \ldots r_{n}^{x_{N}} v_{1}^{\left(x_{1}\right)} \\
& \times\left(q_{1}\right) v_{2}^{\left(x_{2}\right)}\left(q_{2}\right) \ldots v_{N}^{\left(x_{N}\right)}\left(q_{N}\right)
\end{aligned}
$$

and for $q \geq k \geq 1, \Omega=\mathcal{N} \backslash\{i\}, i=1,2, \ldots, N$,

$$
\begin{aligned}
& w_{\Omega}(q, k)=\sum_{\left\{\begin{array}{c}
\sum_{j \neq i} x_{j}=k \\
\sum_{j \neq i} q_{j}=q
\end{array}\right\}} \frac{k !}{x_{1} ! \ldots x_{i-1} ! x_{i+1} ! \ldots x_{N} !} \\
& \times \prod_{j \neq i} r_{j}^{x_{j}} v_{j}^{\left(x_{j}\right)}\left(q_{j}\right)
\end{aligned}
$$

where $\dot{r}_{j}=\lambda_{j} /\left(\lambda_{0}-\lambda_{i}\right)$ for $j \neq i$.

Now, let $\tilde{p}_{0}\left(q, \lambda_{\Omega} z, \Omega\right)$ be the probability that a total of $q$ units are demanded of items in set $\Omega$ in $z$ time units by the customers arriving according to a compound Poisson process with rate $\lambda_{\Omega}\left(=\sum_{i \in \Omega} \lambda_{i}\right)$ and batch size with p.m.f. given by $w_{\Omega}(q, k)$. Then,

$$
\tilde{p}_{0}\left(q, \lambda_{\Omega} z, \Omega\right)=\sum_{k=1}^{q} p_{0}\left(k, \lambda_{\Omega} z\right) w_{\Omega}(q, k) .
$$

The joint probability density function of $Y$ and $Q_{0}$ for the compound Poisson demand case can now be expressed as follows. 
Lemma 4.1:

$$
f_{Y, Q_{0}}(y, q)= \begin{cases}\phi_{0}^{m-1} \tilde{p}_{0}\left(q, \lambda_{\mathcal{N}} T, \mathcal{N}\right) & \text { if } y=m T, m \geq 1,0<q<Q \\ \phi_{0}^{m-1} \sum_{k=0}^{Q-1} \sum_{j=k}^{Q-1} f\left(y-(m-1) T, k+1, \lambda_{0}\right) w_{\mathcal{N}}(j, k)\left[\sum_{i=1}^{N} r_{i} v_{i}(q-j)\right] & \text { if }(m-1) T<y<m T, m \geq 1, q \geq Q\end{cases}
$$

Lemma 4.1 can be used to obtain the marginal distributions of $Y$ and $Q_{0}$ as well as $E[Y]$. Analogous to the unit Poisson demand case, we have the following result:

Proposition 4.1: The steady state p.d.f. of the stochastic process $\xi_{i}(t)$ is given as

$$
g_{i}\left(n_{i}, n_{0}, z\right)=C_{1} \tilde{p}_{0}\left(n_{i}, \lambda_{\{i\}} z,\{i\}\right) \tilde{p}_{0}\left(n_{0}, \lambda_{\{\mathcal{N} \backslash\{i\}\}} z,\{\mathcal{N} \backslash\{i\}\}\right)
$$

for $0<z<T$ and $0 \leq n_{0}+n_{i} \leq Q-1, n_{0} \geq 0, n_{i} \geq$ $0, i=1,2, \ldots, N$ where $C_{1}$ is the normalizing constant given by

$C_{1}$

$=\left[\sum_{n_{0}=0}^{Q-1} \sum_{n_{i}=0}^{Q-1-n_{0}} \int_{z=0}^{T} \tilde{p}_{0}\left(n_{i}, \lambda_{\{i\}} z,\{i\}\right) \tilde{p}_{0}\left(n_{0}, \lambda_{\{\mathcal{M} \backslash\{i\}\}},\{\mathcal{N} \backslash\{i\}\}\right) d z\right]^{-1}$.

From Proposition 4.1 we obtain, as before, the steadystate distribution of item $i$ :

Proposition 4.2:

$$
\begin{aligned}
& \varphi_{i}\left(S_{i}-n_{i}\right) \\
& =C_{1} \sum_{n_{0}=1}^{Q-1-n_{i}} \int_{z=0}^{T} \tilde{p}_{0}\left(n_{i}, \lambda_{\{i\}} z,\{i\}\right) \tilde{p}_{0}\left(n_{0}, \lambda_{\{\mathcal{M} \backslash\{i\}\}} z,\{\mathcal{N} \backslash\{i\}\}\right) d z
\end{aligned}
$$

for $0 \leq n_{i} \leq Q-1, i=1,2, \ldots, N$.

Finally, for $i=1,2, \ldots, N$,

$$
\begin{array}{r}
P\left(O H_{i}=y_{i}\right)=\sum_{n_{i}=S_{i}-Q-1}^{\min \left(S_{i}, y_{i}\right)} \varphi_{i}\left(n_{i}\right) \tilde{p}_{0}\left(n_{i}-y_{i}, \lambda_{\{i\}} L_{i},\{i\}\right) \\
0 \leq y_{i} \leq S_{i} \\
P\left(B O_{i}=y_{i}\right)=\sum_{n_{i}=S_{i}-Q-1}^{S_{i}} \varphi_{i}\left(n_{i}\right) \tilde{p}_{0}\left(n_{i}+y_{i}, \lambda_{\{i\}} L_{i},\{i\}\right) \\
y_{i} \geq 0 .
\end{array}
$$

Note that the results of Lemma 4.1, Propositions 4.1 and 4.2, and the expressions in Eqs. (12) and (13) for the compound Poisson demand case are similar to those given in Lemma 2.1, Propositions 2.1 and 2.2, and the expressions in Eqs. (4) and (5) for the unit Poisson demand case except for the modified probabilities.

Since the $(\mathbf{R}, T)$ and $(Q, \mathbf{S})$ policies are special cases of the $(Q, \mathbf{S}, T)$ policy, the above generalization also provides the compound Poisson demand counterparts of these policies.

\section{NUMERICAL RESULTS}

In this section, we present an extensive numerical study to gain insights about (i) the performance of the proposed policy vis a vis the existing joint replenishment policies, (ii) sensitivity of the optimal policy parameters of the proposed $(Q, \mathbf{S}, T)$ policy with respect to various system parameters, and (iii) the effect of batch demand processes. We begin with a brief discussion of some computational issues.

\subsection{Computational Issues}

Before we proceed with the results of our numerical study, a few words on the behavior of $A C(Q, \mathbf{S}, T)$ and the employed search algorithm are in order.

We first observe that for a given $(Q, T)$ pair, the optimization problem to find $\mathbf{S}^{*}$ can be decomposed into $N$ independent sub-problems in each of which we solve for $S_{i}^{*}$ separately. This separability property greatly reduces the complexity of the optimization problem.

For optimization, we employ exhaustive search over a large solution space. The search space consists of $Q \in$ $\left[Q^{\min }, Q^{\max }\right], T \in\left[T^{\min }, T^{\max }\right], S_{i} \in\left[S_{i}^{\min }, S_{i}^{\max }\right]$ for $i=$ $1,2, \ldots, N$ with increments of $\Delta_{Q}=1, \Delta_{T}=0.01$, $\Delta_{S_{i}}=1$ and the boundaries of the space are given by

$$
\begin{gathered}
Q^{\min }=\max \left(1, Q_{m}\right), Q^{\max }=\max \left(5 Q_{m}, Q_{m}+200\right), \\
\text { where } Q_{m}=\sqrt{2 \lambda_{0}\left(K+\sum_{i=1}^{N} k_{i}\right) / \sum_{i=1}^{N} r_{i} h_{i}} \\
T^{\min }=0.5 Q^{\min } / \lambda_{0}, T^{\max }=1.5 Q^{\max } / \lambda_{0}, \\
S_{i}^{\min }=\min \left(Q_{i}\left[\lambda_{i} L_{i}\right]\right), S_{i}^{\max }=Q_{i}+5\left[\lambda_{i} L_{i}\right], \\
\text { where } Q_{i}=\sqrt{2 \lambda_{i}\left(K r_{i}+k_{i}\right) / h_{i}} .
\end{gathered}
$$


Here, $[x]$ denotes the smallest integer larger than or equal to $x$. The employed algorithm is provided in the Appendix.

In a preliminary study, we also investigated and observed the unimodality of $A C(Q, \mathbf{S}, T)$ through an iterative search algorithm over a broad solution space with randomized initial points. A total of 100 initial points $\hat{Q}$ and $\hat{\mathbf{S}}$ were randomly selected over the following ranges: $\hat{Q} \in[1$, $\left.\max \left(10 Q_{m}, 1000\right)\right]$ and $\hat{S}_{i} \in\left[1, Q_{i}+10\left[\lambda_{i} L_{i}\right]\right]$. One iteration of our iterative search algorithm consisted of three consecutive optimization problems for one of the policy decision variables while keeping the other two constant. The iterative algorithm starts with a randomly selected $\hat{Q}$ and $\hat{\mathbf{S}}$ and ends when either the same policy parameter values are obtained in two consecutive iterations or the number of iterations reaches 1000 . The search space and the increments for unimodality investigation were the same as those given above except that we set $Q_{\min }=\max \left(1, Q_{m}\right), Q^{\max }$ $=\max \left(10 Q_{m}, 1000\right), S_{i}^{\max }=Q_{i}+10\left|\lambda_{i} L_{i}\right|$ to get a larger range.

We observed that the solution of the algorithm converges to the same policy parameter values for all 100 starting points. Incidentally, all convergences occurred in fewer than 1000 iterations and no converged solution occurred on the boundaries of the search space. Clearly, this does not guarantee the global unimodality of $A C(Q, \mathbf{S}, T)$. However, given the very broad range of the starting points and the optimization search space, the observed convergence can be taken as an experimental indication for unimodality.

\subsection{Comparison with Existing Policies}

In this subsection, we examine the efficacy of the proposed control policy. In particular, we examine the cost improvements achieved by the proposed policy and attempt to identify the operational environments in which it is beneficial to implement the proposed policy in lieu of the existing ones in the literature. Note that all of the available models have been developed only for unit demands.

For policy comparisons, we introduce some notation below. We let $A C_{\mathscr{P}}^{*}$ denote the optimal cost rate of a given policy $\mathscr{P}$ where $\mathscr{P}$ can be one of the following: Our proposed $(Q, \mathbf{S}, T)$ policy; $P(\mathbf{s}, \mathbf{S})$ in [33]; $(Q, \mathbf{S})$ in [21] (and [22]; the can-order policies, $(\mathbf{s}, \mathbf{c}, \mathbf{S})_{F}$ and $(\mathbf{s}, \mathbf{c}, \mathbf{S})_{M}$, as calculated in [9] and [18], resp.; and, $Q(\mathbf{s}, \mathbf{S})$ in [20]. Note that we have excluded the $M P$ policy in [3] since it has previously been shown to be inferior to the aforementioned policies in the literature. As a measure of the performance of the proposed $(Q, \mathbf{S}, T)$ policy, we use the percentage improvement $\Delta_{\mathscr{P}} \%$ over policy $\mathscr{P}$ as follows:

$$
\Delta_{\mathscr{P}} \%=\frac{A C_{\mathscr{P}}^{*}-A C_{(Q, \mathbf{S}, T)}^{*}}{A C_{(Q, \mathbf{S}, T)}^{*}} \times 100 .
$$

A positive entry for $\% \Delta_{\mathscr{P}}$, by definition, means that the proposed policy dominates policy $\mathscr{P}$. At this point, a remark on how the $A C_{\mathscr{P}}^{*}$ values are obtained is in order. Among the considered policies, the analyses for the $(Q, \mathbf{S})$ and $M P$ policies in the literature and the proposed $(Q, \mathbf{S}, T)$ policy herein are exact. Therefore, the corresponding $A C_{\mathscr{P}}^{*}$ values are also exact. However, for the inventory systems operating under the $P(\mathbf{s}, \mathbf{S}), Q(\mathbf{s}, \mathbf{S}),(\mathbf{s}, \mathbf{c}, \mathbf{S})_{F}$, and $(\mathbf{s}, \mathbf{c}, \mathbf{S})_{M}$ policies, the models and the corresponding cost functions in the literature are only approximations. Consequently, the best policy parameter values for these policies are obtained only for the approximate cost functions. To compute the corresponding true $A C_{\mathscr{P}}^{*}$ under these policies, one must simulate the inventory systems with the given policy parameter values. The simulation results for $A C_{(\mathbf{s}, \mathbf{c}, \mathbf{S})_{F}}$ and $A C_{(\mathbf{s}, \mathbf{c}, \mathbf{S})_{M}}$ have already been reported in [33] and [18], respectively, and were used directly for our numerical study. For the $Q(\mathbf{s}, \mathbf{S}), P(\mathbf{s}, \mathbf{S})$ policies, we solved for the best policy parameters using the approximate cost functions as developed in [33] and [20] and then simulated the inventory systems operating under these two policies to obtain the corresponding true $A C_{Q(\mathbf{s}, \mathbf{S})}^{*}$ and $A C_{P(\mathbf{s}, \mathbf{S})}^{*}$. For our simulations, we used a run length of 100,000 ordering instances with a warm-up period of 10,000 order placements and 100 replications to obtain the reported average figures.

Our numerical study indicates that the performances of joint replenishment policies and, thereby, the dominance of one over the others depends greatly on the cost and demand rate structures prevalent among the items. Therefore, we present our policy comparisons in two groups.

\subsubsection{Atkins-Iyogun and Viswanathan Test Beds}

For the first part of our policy comparisons, we use two test beds. The first one-the Atkins-Iyogun test bedconsisting of 19 instances, was initially introduced by the authors for their sensitivity study [3] and has subsequently been adopted as the standard test bed for comparison of any proposed stochastic joint replenishment policy. The second one - the Viswanathan test bed-has been developed by the author for comparing the robustness of the $P(\mathbf{s}, \mathbf{S})$ policy against the Atkins-Iyogun policies and considers an extensive set of parameter combinations (120 instances). Both sets consider 12 items. There are no reported results on the performance of $Q(\mathbf{s}, \mathbf{S})$ policy over the Viswanathan test bed in the literature. Hence, our numerical study also provides performance results on this policy for the first time.

Before we proceed with individual comparisons, we present a summary of our findings over all experiment instances (139 total) in the Atkins-Iyogun and Viswanathan sets. We observed that the proposed policy is the best policy in 100 of 139 instances with an average improvement of $1.14 \%$ and the maximum improvement of $3.55 \%$ over the 
Table 1. Performance of $(Q, \mathbf{S}, T)$ policy for the 12-item problem set.

\begin{tabular}{|c|c|c|c|c|c|c|}
\hline \multicolumn{2}{|c|}{ Problem parameters } & $A C_{(Q, \mathbf{S}, T)}$ & $\Delta_{P(\mathbf{s}, \mathbf{S})} \%$ & $\Delta_{(Q, \mathbf{S})} \%$ & $\Delta_{Q(\mathbf{s}, \mathbf{S})} \%$ & $\Delta_{(\mathbf{s}, \mathbf{c}, \mathbf{S})_{M}} \%$ \\
\hline \multirow{5}{*}{$\pi=30, \rho=0, h=2$} & $K=50$ & 1109.90 & 1.01 & 5.60 & 0.27 & -0.09 \\
\hline & $K=100$ & 1174.21 & 0.91 & 3.22 & 0.25 & 3.15 \\
\hline & $K=150$ & 1234.12 & 0.56 & 1.37 & -0.24 & 4.46 \\
\hline & $K=200$ & 1282.29 & 0.22 & 0.45 & -0.58 & 5.69 \\
\hline & $K=250$ & 1323.02 & 0.31 & 0.00 & -0.46 & 6.58 \\
\hline$\pi=30, \rho=0, h=6$ & $K=150$ & 2279.97 & -0.58 & 1.05 & -1.22 & 1.45 \\
\hline \multirow[t]{5}{*}{$\pi=0, \rho=30, h=2$} & $K=20$ & 878.91 & 0.82 & 8.54 & 0.36 & -0.80 \\
\hline & $K=50$ & 928.40 & 0.59 & 5.34 & 0.07 & 1.72 \\
\hline & $K=100$ & 990.02 & 0.80 & 2.62 & -0.40 & 4.44 \\
\hline & $K=150$ & 1044.04 & -0.11 & 0.76 & -0.77 & 5.94 \\
\hline & $K=200$ & 1087.17 & -0.21 & 0.02 & -0.84 & 6.92 \\
\hline \multirow[t]{3}{*}{$\pi=0, \rho=30, h=6$} & $K=100$ & 1635.98 & -0.79 & 2.39 & -1.29 & 1.47 \\
\hline & $K=150$ & 1717.94 & -0.70 & 0.82 & -1.23 & 1.69 \\
\hline & $K=200$ & 1786.89 & -0.51 & 0.01 & -1.07 & 1.90 \\
\hline \multirow[t]{5}{*}{$\pi=0, \rho=30, h=20$} & $K=20$ & 2294.78 & 1.33 & 10.12 & 5.93 & 0.83 \\
\hline & $K=50$ & 2395.45 & 1.33 & 7.70 & 4.67 & 1.63 \\
\hline & $K=100$ & 2533.82 & 1.10 & 5.34 & 3.57 & 1.46 \\
\hline & $K=150$ & 2739.46 & -2.02 & 0.49 & 3.52 & -1.35 \\
\hline & $K=200$ & 2721.67 & 2.59 & 4.13 & 3.48 & 3.60 \\
\hline
\end{tabular}

next best policy in these instances. In the remaining 39 cases, $Q(\mathbf{s}, \mathbf{S})$ is the best policy in $24 ; P(\mathbf{s}, \mathbf{S})$ is the best policy in 8; and $(\mathbf{s}, \mathbf{c}, \mathbf{S})_{M}$ is the best policy in 7 instances. We never see $M P,(Q, \mathbf{S})$, and $(\mathbf{s}, \mathbf{c}, \mathbf{S})_{F}$ to be the best policy.

Next, we discuss our findings for each test bed separately, beginning with the Atkins-Iyogun test bed. This set consists of 12 items; the items have identical shortage and unit holding costs but differ in their item-specific ordering costs, demand rates, and delivery lead times. The item-specific costs are as follows: $k_{i}=\{10,10,20,20,40,20,40,40$, $60,60,80,80\}$, the demand rates are given by $\lambda_{i}=\{40$, $35,40,40,40,20,20,20,28,20,20,20\}$, and the lead times are taken as $L_{i}=\{0.2,0.5,0.2,0.1,0.2,1.5,1.0$, $1.0,1.0,1.0,1.0,1.0,1.0\}$ for $i=1, \ldots, 12$. We tabulate the problem parameters common to all items in Table 1. In Table 1 we also report the corresponding $A C_{(Q, \mathbf{S}, T)}^{*}$ and $\% \Delta_{\mathscr{P}}$ under the four policies considered.

The dominance of the proposed policy is not monotone across the experiment instances. The $(Q, \mathbf{S}, T)$ policy performs better than all other existing policies in 6 of 19 experiment instances.

For the remaining 13 experiment instances, it is dominated in 10 cases by $Q(\mathbf{s}, \mathbf{S})$, twice by $(\mathbf{s}, \mathbf{c}, \mathbf{S})_{M}$, and once by $P(\mathbf{s}, \mathbf{S})$. We see that the $(Q, \mathbf{S})$ policy is never the best policy. Across the entire Atkins-Iyogun set, the average savings achieved through the implementation of the proposed policy in lieu of each of the existing policies are as follows: $0.35 \%$ over $P(\mathbf{s}, \mathbf{S}), 3.39 \%$ over $(Q, \mathbf{S}), 0.74 \%$ over $Q(\mathbf{s}, \mathbf{S})$, and $2.65 \%$ over $(\mathbf{s}, \mathbf{c}, \mathbf{S})_{M}$.

In the instances where the proposed policy gives the best solution, the average improvement over the next best policy is $1.03 \%$.
It is interesting to note that the $(Q, \mathbf{S})$ policy performs so poorly with an average underperformance of $3.39 \%$ compared to the proposed policy. With the incorporation of the time trigger, i.e., increasing the dimensionality by one, we achieve significant improvements

Another interesting (untabulated) observation is that $(Q$, S, T) has, in all instances, resulted in a higher optimal system fill rate than the other policies. Especially, $(Q, \mathbf{S})$ and $\mathbf{Q}(\mathbf{s}, \mathbf{S})$ policies have resulted in significantly lower optimal system fill rates. Although not considered herein, this may have important implications for inventory settings with non-linear shortage costs.

The performance of the proposed policy is somewhat mixed over the cost parameter set; a clear dominance region is not discernible. However, a general observation is that the proposed policy performs best for lower shortage, higher holding, and lower common ordering costs. These parameter values also correspond to the cases where pro-active ordering (i.e., placing the orders at review epochs) becomes the dominant reordering mode, explaining the advantageousness of the policy.

The second data set used in policy comparison is the one generated by Viswanathan [33]. For this set, the demand rates, lead times, and item-specific ordering costs are retained as in the 12-item problem set of Atkins and Iyogun; and different values are considered for the remaining costs as follows: $\pi=0, K \in\{20,50,100,200,500\}, h \in$ $\{2,6,10,200,600,1000\}$, and $\rho \in\{10,50,100,1000$, $5000,10000,20000\}$. The considered instances and the results are tabulated in Tables 2 and 3. (We note that comparison with $(\mathbf{s}, \mathbf{c}, \mathbf{S})_{M}$ has been made for the 36 instances reported in the study by Melchiors [18] to ensure 
Table 2. Performance of $(Q, \mathbf{S}, T)$ policy for the 12-item problem set.

\begin{tabular}{|c|c|c|c|c|c|c|c|c|c|c|c|c|c|c|c|}
\hline$K$ & $\rho$ & $h$ & $A C_{(Q, S, T)}$ & $\Delta_{P(s, S)} \%$ & $\Delta_{(Q, S)} \%$ & $\Delta_{(s, S)} \%$ & $\Delta_{(s, c, S)_{M}} \%$ & $K$ & $\rho$ & $h$ & $A C_{(Q, S, T)}$ & $\Delta_{P(s, S)} \%$ & $\Delta_{(Q, S)} \%$ & $\Delta_{Q(s, S)} \%$ & $\Delta_{(s, c, S)_{M}} \%$ \\
\hline \multirow[t]{12}{*}{20} & 10 & 2 & 772 & 0.79 & 8.34 & 0.51 & - & \multirow[t]{3}{*}{50} & \multirow[t]{3}{*}{10} & 2 & 810 & 1.11 & 5.96 & 0.61 & - \\
\hline & & 6 & 1176 & -0.84 & 7.08 & 1.97 & - & & & 6 & 1221 & 0.07 & 5.69 & 1.98 & - \\
\hline & & 10 & 1401 & $-\mathbf{3 . 1 3}$ & 4.70 & 3.72 & - & & & 10 & 1443 & -1.58 & 4.21 & 3.67 & - \\
\hline & \multirow[t]{3}{*}{50} & 2 & 905 & 2.66 & 10.66 & 2.00 & - & & \multirow[t]{3}{*}{50} & 2 & 954 & 2.61 & 7.44 & 1.98 & - \\
\hline & & 6 & 1587 & -0.57 & 7.79 & -1.02 & - & & & 6 & 1669 & -0.72 & 4.84 & -1.20 & - \\
\hline & & 10 & 1918 & 3.82 & 12.84 & 3.56 & - & & & 10 & 2008 & 3.92 & 10.17 & 3.38 & - \\
\hline & \multirow[t]{3}{*}{100} & 2 & 965 & 1.67 & 9.79 & 0.74 & - & & \multirow[t]{3}{*}{100} & 2 & 1021 & 1.26 & 6.13 & 0.70 & - \\
\hline & & 6 & 1727 & -0.63 & 8.60 & -0.94 & - & & & 6 & 1821 & -0.49 & 6.27 & -1.04 & - \\
\hline & & 10 & 2169 & 2.41 & 12.02 & 2.16 & - & & & 10 & 2276 & 2.72 & 8.99 & 2.20 & - \\
\hline & \multirow[t]{3}{*}{200} & 2 & 1008 & 1.99 & 10.35 & 0.70 & - & & \multirow[t]{3}{*}{200} & 2 & 1068 & 1.39 & 6.58 & 0.67 & - \\
\hline & & 6 & 1854 & -0.54 & 9.21 & -0.87 & - & & & 6 & 1955 & -0.15 & 5.74 & -0.83 & - \\
\hline & & 10 & 2398 & 1.55 & 11.49 & 1.20 & - & & & 10 & 2522 & 1.82 & 8.14 & 1.23 & - \\
\hline \multirow[t]{12}{*}{100} & \multirow[t]{3}{*}{10} & 2 & 863 & 0.94 & 3.45 & 0.47 & - & \multirow[t]{6}{*}{200} & \multirow[t]{3}{*}{10} & 2 & 948 & 0.74 & 1.24 & 0.20 & - \\
\hline & & 6 & 1301 & -0.07 & 3.12 & 1.15 & - & & & 6 & 1418 & 0.72 & 1.51 & 0.92 & - \\
\hline & & 10 & 1523 & -0.91 & 2.65 & 2.57 & - & & & 10 & 1648 & 0.49 & 1.49 & 1.51 & - \\
\hline & \multirow[t]{3}{*}{50} & 2 & 1023 & 1.75 & 4.00 & 1.18 & - & & \multirow[t]{3}{*}{50} & 2 & 1118 & 1.61 & 1.81 & 0.82 & - \\
\hline & & 6 & 1770 & -0.73 & 2.34 & -1.30 & - & & & 6 & 1933 & -0.62 & 0.22 & -1.29 & - \\
\hline & & 10 & 2129 & 3.61 & 7.35 & 3.11 & - & & & 10 & 2355 & 2.37 & 3.20 & 1.79 & - \\
\hline & \multirow{3}{*}{100} & 2 & 1085 & 1.37 & 3.61 & 0.66 & - & & \multirow[t]{3}{*}{100} & 2 & 1181 & 1.53 & 1.63 & 0.69 & - \\
\hline & & 6 & 1932 & -0.51 & 2.57 & -1.20 & - & & & 6 & 2116 & -0.91 & 0.50 & -1.66 & - \\
\hline & & 10 & 2406 & 2.79 & 6.59 & 2.12 & - & & & 10 & 2635 & 2.20 & 3.02 & 1.57 & - \\
\hline & \multirow[t]{3}{*}{200} & 2 & 1137 & 1.32 & 3.65 & 0.62 & - & & \multirow[t]{3}{*}{200} & 2 & 1237 & 1.46 & 1.56 & 0.49 & - \\
\hline & & 6 & 2079 & -0.42 & 2.65 & -1.20 & - & & & 6 & 2269 & -0.62 & 0.32 & -1.46 & - \\
\hline & & 10 & 2665 & 1.89 & 5.54 & 1.08 & - & & & 10 & 2899 & 1.86 & 2.47 & 0.94 & - \\
\hline \multirow[t]{6}{*}{500} & \multirow[t]{3}{*}{10} & 2 & 1133 & 0.45 & 0.04 & 0.00 & - & \multirow[t]{6}{*}{500} & \multirow[t]{3}{*}{100} & 2 & 1396 & 0.93 & 0.02 & -0.07 & - \\
\hline & & 6 & 1696 & 0.25 & 0.04 & -0.06 & - & & & 6 & 2428 & 0.82 & 0.02 & 0.00 & - \\
\hline & & 10 & 1966 & 0.19 & 0.00 & 0.00 & - & & & 10 & 3115 & 0.71 & 0.00 & 0.00 & - \\
\hline & \multirow[t]{3}{*}{50} & 2 & 1329 & 0.74 & 0.05 & 0.00 & - & & \multirow[t]{3}{*}{200} & 2 & 1457 & 0.96 & 0.05 & 0.00 & - \\
\hline & & 6 & 2238 & 0.70 & 0.01 & 0.00 & - & & & 6 & 2597 & 1.00 & 0.09 & 0.00 & - \\
\hline & & 10 & 2806 & 0.62 & 0.00 & 0.00 & - & & & 10 & 3389 & 0.91 & 0.01 & 0.00 & - \\
\hline
\end{tabular}

fairness in comparing simulation-based results for the latter.)

The $(Q, \mathbf{S}, T)$ policy performs better than all other existing policies in 94 of 120 experiment instances. For the remaining 26 experiment instances, it is dominated in 14 cases by $Q(\mathbf{s}, \mathbf{S}), 7$ times by $P(\mathbf{s}, \mathbf{S})$, and 5 times by $(\mathbf{s}, \mathbf{c}$, $\mathbf{S})_{M}$. As in the Atkins-Iyogun set, $(Q, \mathbf{S})$ is never the best policy.

Over all 120 experiment instances, the average savings achieved through the implementation of the proposed policy in lieu of each of the existing policies are as follows: $1.25 \%$ over $P(\mathbf{s}, \mathbf{S}), 4.16 \%$ over $(Q, \mathbf{S})$ and $1.07 \%$ over $Q(\mathbf{s}, \mathbf{S})$, and $0.82 \%$ over $(\mathbf{s}, \mathbf{c}, \mathbf{S})_{M}$.

As in the Atkins-Iyogun set, the dominance of the proposed policy is not monotone across the experiment instances. However, in the cases where the proposed policy gives the best solution, the improvement over the next best policy is $1.13 \%$. Over these 94 instances, the maximum saving was observed to be $3.55 \%$.

To give a broader view of the policy performances, comparison summaries are presented in two tables: Tables 4 and 5 . In both tables, we have included summaries of the unreported results on $M P$ and $(\mathbf{s}, \mathbf{c}, \mathbf{S})_{F}$ as well.
In Table 4 we provide a pairwise comparison in a matrix format across instances where one policy dominates the other. The first column lists the policies in the chronological order in which they have been proposed in the literature; the second column reports the number of control parameters that a particular policy employs for the standard test bed of 12 items. Each element of the matrix reports two entities: the average improvement in the expected total cost rate achieved by policy $\mathscr{P}_{i}$ over policy $\mathscr{P}_{j}$ in the experiment instances where $\mathscr{P}_{i}$ dominates $\mathscr{P}_{j}$; and the number of such instances in parentheses. The first row of Table 4 gives the performance of the proposed policy in comparison with the other policies. For example, we see that $(Q, \mathbf{S}, T)$ dominates $Q(\mathbf{s}, \mathbf{S})$ in 115 of 139 considered instances; and the average improvement in such instances achieved over $Q(\mathbf{s}$, $\mathbf{S})$ is $1.43 \%$. Similarly, the proposed policy is better than (s, c, S $)_{M}$ with an average improvement of $1.85 \%$ in 47 of 55 considered instances, and so on and so forth.

In Table 5, we provide an overall comparison of the average performance of the policies. In the same format as before, we list the policies in the chronological order, the dimension of each policy and present the average percentage change in the expected total cost rate under policy $P_{i}$ 
Table 3. Performance of $(Q, \mathbf{S}, T)$ policy for the 12-item problem set.

\begin{tabular}{|c|c|c|c|c|c|c|c|c|c|c|c|c|c|c|c|}
\hline$K$ & $\rho$ & $h A$ & $C_{(Q, \mathbf{S}, T)}$ & $\Delta_{P(\mathbf{s}, \mathbf{S})} \%$ & $\Delta_{(Q, \mathbf{S})} \%$ & $\Delta_{(\mathbf{s}, \mathbf{c}, \mathbf{S})_{M}} \%$ & $\% \quad K$ & $\rho$ & $h A$ & $C_{(Q, \mathbf{s}, T)}$ & $\Delta_{P * \mathbf{s}, \mathbf{s})} \%$ & $\Delta_{(Q, \mathbf{S})} \%$ & $\Delta_{Q(\mathbf{s}, \mathbf{s})} \%$ & $\Delta_{(\mathbf{s}, \mathbf{c}, \mathbf{S})_{M}} \%$ & \\
\hline 20 & 1000 & 200 & 18175 & 2.56 & 9.22 & 2.44 & 1.96 & 50 & 1000 & 200 & 18627 & 2.74 & 7.87 & 2.39 & - \\
\hline & & 600 & 34210 & 0.01 & 4.90 & 2.54 & 0.30 & & & 600 & 34597 & 0.74 & 4.46 & 2.36 & - \\
\hline & & 1000 & 44510 & -2.36 & 1.55 & 1.11 & -2.85 & & & 1000 & 44964 & -1.67 & 1.18 & 0.77 & - \\
\hline & 5000 & 200 & 25501 & 3.78 & 10.02 & 3.53 & 2.80 & & 5000 & 200 & 26076 & 3.85 & 8.53 & 3.43 & - \\
\hline & & 600 & 56828 & 1.61 & 5.56 & 1.26 & 0.96 & & & 600 & 57532 & 1.62 & 4.99 & 1.34 & - \\
\hline & & 1000 & 78690 & 3.07 & 5.95 & 2.79 & 2.42 & & & 1000 & 79523 & 3.11 & 5.39 & 2.88 & - \\
\hline & 10000 & 200 & 28575 & 3.36 & 9.47 & 3.11 & 2.48 & & 10000 & 200 & 29156 & 3.48 & 8.04 & 3.08 & - \\
\hline & & 600 & 67081 & 0.75 & 4.08 & 0.31 & 0.04 & & & 600 & 67910 & 0.70 & 3.21 & 0.33 & - \\
\hline & & 1000 & 96323 & 1.50 & 3.92 & 1.12 & 0.76 & & & 1000 & 97329 & 1.46 & 3.40 & 1.12 & - \\
\hline & 20000 & 200 & 31789 & 2.01 & 7.72 & 1.76 & 1.09 & & 20000 & 200 & 32436 & 2.01 & 6.38 & 1.60 & - \\
\hline & & 600 & 76249 & 0.89 & 3.90 & 0.29 & -0.04 & & & 600 & 77131 & 0.86 & 3.17 & 0.33 & - \\
\hline & & 1000 & 112118 & 1.42 & 3.54 & 1.01 & 0.74 & & & 1000 & 113019 & 1.53 & 3.27 & 1.17 & - \\
\hline 100 & 1000 & 200 & 19198 & 2.47 & 6.28 & 2.03 & 1.69 & 200 & 1000 & 200 & 19999 & 2.59 & 4.83 & 1.96 & - \\
\hline & & 600 & 35137 & 1.32 & 4.15 & 2.16 & 0.67 & & & 600 & 36059 & 1.65 & 3.47 & 2.06 & - \\
\hline & & 1000 & 45423 & -0.99 & 1.19 & 0.99 & -1.48 & & & 1000 & 46270 & -0.11 & 1.18 & 0.93 & - \\
\hline & 5000 & 200 & 26781 & 3.75 & 7.08 & 3.09 & 2.43 & & 5000 & 200 & 27772 & 3.56 & 5.54 & 2.89 & - \\
\hline & & 600 & 58589 & 1.50 & 3.63 & 1.11 & 0.87 & & & 600 & 60123 & 1.43 & 2.76 & 0.95 & - \\
\hline & & 1000 & 81123 & 2.56 & 4.20 & 2.21 & 1.84 & & & 1000 & 82893 & 2.61 & 3.42 & 2.05 & - \\
\hline & 10000 & 200 & 29981 & 3.10 & 6.49 & 2.54 & 1.91 & & 10000 & 200 & 31019 & 3.20 & 4.96 & 2.46 & - \\
\hline & & 600 & 68965 & 0.70 & 2.51 & 0.25 & 0.00 & & & 600 & 70541 & 0.64 & 1.93 & 0.18 & - \\
\hline & & 1000 & 98678 & 1.38 & 2.91 & 1.04 & 0.68 & & & 1000 & 100627 & 1.45 & 2.05 & 0.94 & - \\
\hline & 20000 & 200 & 33220 & 1.94 & 5.00 & 1.44 & 0.54 & & 20000 & 200 & 34323 & 2.08 & 3.73 & 1.33 & - \\
\hline & & 600 & 78346 & 0.74 & 2.35 & 0.21 & -0.02 & & & 600 & 80045 & 0.64 & 1.66 & 0.20 & - \\
\hline & & 1000 & 114420 & 1.55 & 2.88 & 1.12 & 0.81 & & & 1000 & 116708 & 1.32 & 2.13 & 0.91 & - \\
\hline 500 & 1000 & 200 & 21917 & 1.83 & 2.33 & 1.18 & 1.40 & 500 & 10000 & 200 & 33390 & 2.77 & 2.86 & 1.89 & 1.37 \\
\hline & & 600 & 38526 & 1.54 & 1.96 & 1.24 & 1.25 & & & 600 & 74001 & 0.87 & 0.86 & 0.08 & -0.06 \\
\hline & & 1000 & 48581 & 0.80 & 1.20 & 1.02 & 0.50 & & & 1000 & 104610 & 1.49 & 1.58 & 0.80 & 0.52 \\
\hline & 5000 & 200 & 30128 & 2.80 & 3.11 & 1.92 & 1.54 & & 20000 & 200 & 36815 & 1.76 & 1.96 & 0.85 & 0.19 \\
\hline & & 600 & 63345 & 1.48 & 1.57 & 0.77 & 0.68 & & & 600 & 83590 & 0.80 & 0.91 & 0.18 & 0.24 \\
\hline & & 1000 & 87051 & 1.96 & 2.28 & 1.31 & 1.16 & & & 1000 & 121234 & 1.24 & 1.43 & 0.67 & 0.30 \\
\hline
\end{tabular}

versus $P_{j}$. Note that in creating Table 5, we consider all of the experiment instances, where $P_{i}$ may or may not dominate $P_{j}$. Hence, we have negative averages for certain pairs. A positive entry indicates that policy $P_{i}$ provides that much average percentage improvement in the cost rate over $P_{j}$. A negative entry indicates that the performance of $P_{i}$ is inferior by that much, on average, in comparison with $P_{j}$. The first row gives the performance of the proposed policy ( $Q$, $\mathbf{S}, T)$ with respect to the existing policies. Overall, we see that $(Q, \mathbf{S}, T)$ achieves an improvement of $1.03 \%$ over $Q(\mathbf{s}$, S), $1.46 \%$ over $(\mathbf{s}, \mathbf{c}, \mathbf{S})_{M}, 1.13 \%$ over $P(\mathbf{s}, \mathbf{S}), 4.05 \%$ over $(Q, \mathbf{S}), 4.90 \%$ over $M P$, and $10.25 \%$ over $(\mathbf{s}, \mathbf{c}, \mathbf{S})_{F}$.
When viewing these statistics, one should bear in mind a couple of issues. First, the comparisons are between policies that have already been demonstrated to perform well. The chronological listing enables one to see the evolution of the performances of the policies studied over time, as well. Second, in multi-item settings, the total system costs are substantial in nominal terms; hence, expressing improvements in percentages inevitably understates their impact. Especially in operating environments where margins are known to be notoriously low, as in retail industry, an improvement of even a couple of percentage points does have a substantial impact on profitability (e.g., [11]). In particu-

Table 4. The summary comparison of policies over Atkins-Iyogun and Viswanathan sets across pairwise dominated instances.

\begin{tabular}{|c|c|c|c|c|c|c|c|c|}
\hline Policy & Dimensionality & $(Q, \mathbf{S}, T)$ & $Q(\mathbf{s}, \mathbf{S})$ & $(\mathbf{s}, \mathbf{c}, \mathbf{S})_{M}^{\dagger}$ & $P(\mathbf{s}, \mathbf{S})$ & $(Q, \mathbf{S})$ & $M P$ & $(\mathbf{s}, \mathbf{c}, \mathbf{S})_{F}$ \\
\hline$(Q, \mathbf{S}, T)$ & 14 & - & $1.43(115)$ & $1.85(47)$ & $1.63(111)$ & 4.05 (139) & 4.94 (138) & $10.59(135)$ \\
\hline$Q(\mathbf{s}, \mathbf{S})$ & 25 & $0.94(24)$ & 一 & 3.57 (17) & 0.57 (117) & 2.97 (139) & 3.80 (139) & $10.08(122)$ \\
\hline$(\mathbf{s}, \mathbf{c}, \mathbf{S})_{M}^{\mathrm{a}}$ & 36 & $0.85(8)$ & $1.01(38)$ & - & $0.83(34)$ & $3.39(46)$ & $5.67(44)$ & $7.25(55)$ \\
\hline$P(\mathbf{s}, \mathbf{S})$ & 25 & $0.85(28)$ & $2.48(22)$ & $2.66(21)$ & - & $3.25(125)$ & 3.70 (139) & 10.78 (117) \\
\hline$(Q, \mathbf{S})$ & 13 & $-(0)$ & $-(0)$ & $3.36(9)$ & 0.59 (14) & - & $2.38(91)$ & $12.85(83)$ \\
\hline$M P$ & 24 & 0.20 & $-(0)$ & $2.91(11)$ & $-(0)$ & $2.03(48)$ & - & $12.49(79)$ \\
\hline$(\mathbf{s}, \mathbf{c}, \mathbf{S})_{F}$ & 36 & $1.20(4)$ & $0.87(17)$ & $-(0)$ & $0.42(21)$ & $3.65(56)$ & $4.12(60)$ & - \\
\hline
\end{tabular}

$\mathrm{a}(\mathbf{s}, \mathbf{c}, \mathbf{S})_{M}$ is compared over 55 total instances. 
Table 5. The overall average performance of policies over Atkins-Iyogun and Viswanathan sets across all instances.

\begin{tabular}{|c|c|c|c|c|c|c|c|c|}
\hline Policy & Dimensionality & $(Q, \mathbf{S}, T)$ & $Q(\mathbf{s}, \mathbf{S})$ & $(\mathbf{s}, \mathbf{c}, \mathbf{S})_{M}^{\dagger}$ & $P(\mathbf{s}, \mathbf{S})$ & $\stackrel{(\underset{S}{Q})}{\mathbf{S}}$ & $M P$ & $(\mathbf{s}, \mathbf{c}, \mathbf{S})_{F}$ \\
\hline$(Q, \mathbf{S}, T)$ & 14 & - & 1.03 & 1.46 & 1.13 & 4.05 & 4.90 & 10.25 \\
\hline$Q(\mathbf{s}, \mathbf{S})$ & 25 & -1.00 & - & 0.32 & 0.12 & 2.97 & 3.80 & 9.22 \\
\hline$(\mathbf{s}, \mathbf{c}, \mathbf{S})_{M}{ }^{\mathrm{a}}$ & 36 & -1.41 & -0.25 & - & -0.27 & 2.29 & 3.96 & 7.25 \\
\hline$P(\mathbf{s}, \mathbf{S})$ & 25 & -1.10 & -0.10 & 0.31 & - & 2.87 & 3.70 & 9.12 \\
\hline$(Q, \mathbf{S})$ & 13 & -3.81 & -2.83 & -2.12 & -2.73 & - & 0.86 & 6.20 \\
\hline$M P$ & 24 & -4.59 & -3.62 & -3.94 & -3.50 & -0.77 & - & 5.32 \\
\hline$(\mathbf{s}, \mathbf{c}, \mathbf{S})_{F}$ & 36 & -8.65 & -7.74 & -6.37 & -7.66 & -4.83 & -4.07 & - \\
\hline
\end{tabular}

${ }^{\mathrm{a}}(\mathbf{s}, \mathbf{c}, \mathbf{S})_{M}$ is compared over 55 total instances.

lar, take the case of a major home-improvement retailer with a pretax profit margin (ROA) of $2.7 \%$. If this company could cut its inventory related costs by just $3 \%$, its pretax profits would increase $37 \%$, and the pretax profit margin would rise to nearly $8 \%$. Therefore, the improvements that the proposed policy $(Q, \mathbf{S}, T)$ policy achieves over the existing ones are comparably significant. Moreover, the proposed policy attains such performance levels with parsimony-compare $N+2$ policy parameters of $(Q, \mathbf{S}, T)$ versus $2 N+1$ of $Q(\mathbf{s}, \mathbf{S})$ and $P(\mathbf{s}, \mathbf{S})$ or $3 N$ of the can-order policies. This low dimensionality reduces the computational effort in optimization enormously and eases implementation in practice greatly. Viewing the comparisons in this broader perspective, we conclude that the proposed policy performs well with respect to the existing policies and that this performance is robust over a broad range of environmental parameters.

An aspect of stochastic joint replenishment that has not been studied in the literature before is the impact of the overall system demand rate and of the diversity of demand rates among items. In the next subsection, we focus on such demand rate effects.

\subsubsection{Impact of Demand Rates}

To examine the effects of system or item demand rates, we constructed our own test bed with insights from the Atkins-Iyogun set. (We considered a large number of parameter settings but, for brevity, report only the representative cases.) Since we have identified $Q(\mathbf{s}, \mathbf{S})$ and $P(\mathbf{s}, \mathbf{S})$ policies as the only viable alternatives to our proposed policy in the above comparisons, we compared $(Q, \mathbf{S}, T)$ with only those two and $(Q, \mathbf{S})$ as a special case in this part of our numerical study. We begin with the effect of system demand rate on the performance of the control policies.

We consider $N=8$ identical items with $K=150, h_{i}=$ $h=6, \pi_{i}=\pi=30, \rho_{i}=\rho=0$, and $L_{i}=L=0.2$ and $k_{i}=k=\{0,20,40,60\}$ for all $i$. With identical item demand rates, we consider the system demand rates as $\lambda_{0}=$ $\{160,320,480,640\}$. We present our results in Table 6 .
In all instances, the proposed policy dominates the existing policies. The average savings achieved through the implementation of the proposed policy in lieu of each of the existing policies are as follows: $2.19 \%$ over $P(\mathbf{s}, \mathbf{S})$ and $1.43 \%$ over $(Q, \mathbf{S})$ and $Q(\mathbf{s}, \mathbf{S})$. There is not any discernible difference between the performances of $(Q, \mathbf{S})$ and $Q(\mathbf{s}, \mathbf{S})$. As system demand rate increases, the performances of the policies become somewhat alike.

Next, we examine the effect of item demand rates while keeping the system demand rate constant. This is equivalent to examining the effect of number of items that are jointly replenished for a given system demand rate. Hence, we consider the set of $N$ identical items with $\lambda_{0}=320, K=$ $150, k_{i}=k=20, h_{i}=h=6, \pi_{i}=\pi=30, \rho_{i}=\rho=$ 0 for all $i=1, \ldots, N$. We vary the number of items and lead times as $N=2,4,6,8,10,12$ and $L_{i}=L=0.2$, $0.4,0.6$. Note that individual demand rates are also equal to each other in this set. The results are tabulated in Table 7.

In all cases, the proposed policy dominates the other policies. The average savings achieved through the implementation of the proposed policy in lieu of each of the

Table 6. Performance of $(Q, \mathbf{S}, T)$ policy for identical items with different demand rates and minor ordering cost, $N=8, K=$ $150, L=0.2, h=6, \pi=30, \rho=0$.

\begin{tabular}{cccccc}
\hline$k$ & $\lambda$ & $A C_{(Q, \mathbf{S}, T)}$ & $\Delta_{P *(\mathbf{s}, \mathbf{S})} \%$ & $\Delta_{(Q, \mathbf{S})} \%$ & $\Delta_{Q(\mathbf{s}, \mathbf{S})} \%$ \\
\hline 0 & 20 & $\mathbf{8 3 1 . 9 0}$ & 4.67 & 4.01 & 4.01 \\
& 40 & $\mathbf{1 1 7 7 . 3 8}$ & 3.24 & 3.56 & 3.55 \\
& 60 & $\mathbf{1 4 4 6 . 1 8}$ & 2.17 & 2.18 & 2.19 \\
& 80 & $\mathbf{1 6 7 7 . 4 3}$ & 1.26 & 0.92 & 0.92 \\
20 & 20 & $\mathbf{1 0 5 9 . 8 6}$ & 4.81 & 3.65 & 3.60 \\
& 40 & $\mathbf{1 5 0 2 . 7 8}$ & 3.79 & 1.18 & 1.17 \\
& 60 & $\mathbf{1 8 5 8 . 1 8}$ & 2.13 & 1.15 & 1.15 \\
& 80 & $\mathbf{2 1 5 6 . 1 4}$ & 1.17 & 0.06 & 0.06 \\
40 & 20 & $\mathbf{1 2 5 0 . 4 7}$ & 3.17 & 1.25 & 1.24 \\
& 40 & $\mathbf{1 7 7 5 . 3 7}$ & 2.27 & 1.05 & 1.05 \\
& 60 & $\mathbf{2 1 7 8 . 5 0}$ & 1.64 & 0.98 & 0.99 \\
& 80 & $\mathbf{2 5 2 3 . 7 0}$ & 0.99 & 0.72 & 0.72 \\
60 & 20 & $\mathbf{1 4 0 9 . 0 7}$ & 2.17 & 1.05 & 1.05 \\
& 40 & $\mathbf{2 0 1 2 . 2 4}$ & 0.91 & 0.91 & 0.92 \\
& 60 & $\mathbf{2 4 6 8 . 6 2}$ & 0.41 & 0.18 & 0.18 \\
& 80 & $\mathbf{2 8 4 7 . 5 0}$ & 0.24 & 0.14 & 0.13 \\
\hline
\end{tabular}

Naval Research Logistics DOI 10.1002/nav 
Table 7. Performance of $(Q, \mathbf{S}, T)$ policy for identical items with different lead-time and number of items, $\lambda_{0}=320, K=$ $150, k=20, h=6, \pi=30, \rho=0$.

\begin{tabular}{rrrccc}
\hline$L$ & $N$ & $A C_{(Q, \mathbf{S}, T)}$ & $\Delta_{P(\mathbf{s}, \mathbf{S})} \%$ & $\Delta_{(Q, \mathbf{S})} \%$ & $\Delta_{Q(\mathbf{s}, \mathbf{S})} \%$ \\
\hline 0.2 & 2 & $\mathbf{1 0 5 8 . 9 1}$ & 6.24 & 5.21 & 5.18 \\
& 4 & $\mathbf{1 1 9 1 . 0 8}$ & 5.11 & 3.58 & 3.57 \\
& 6 & $\mathbf{1 3 5 8 . 0 1}$ & 4.34 & 1.86 & 1.84 \\
& 8 & $\mathbf{1 5 0 2 . 7 8}$ & 3.79 & 1.18 & 1.18 \\
& 10 & $\mathbf{1 6 8 1 . 2 9}$ & 1.71 & 0.46 & 0.46 \\
& 12 & $\mathbf{1 8 6 1 . 1 9}$ & 1.60 & 0.32 & 0.33 \\
0.4 & 2 & $\mathbf{1 1 3 0 . 2 1}$ & 5.87 & 4.60 & 4.60 \\
& 4 & $\mathbf{1 3 1 9 . 6 0}$ & 4.85 & 3.26 & 3.26 \\
& 6 & $\mathbf{1 4 9 2 . 2 3}$ & 4.65 & 2.94 & 2.93 \\
& 8 & $\mathbf{1 5 5 0 . 2 1}$ & 4.20 & 2.83 & 2.82 \\
& 10 & $\mathbf{1 7 6 0 . 8 1}$ & 1.14 & 0.38 & 0.38 \\
& 12 & $\mathbf{1 9 1 8 . 5 0}$ & 0.29 & 0.09 & 0.08 \\
0.6 & 2 & $\mathbf{1 1 9 3 . 2 0}$ & 5.59 & 4.67 & 4.67 \\
& 4 & $\mathbf{1 2 7 1 . 1 0}$ & 5.03 & 3.39 & 3.38 \\
& 6 & $\mathbf{1 5 2 4 . 1 0}$ & 4.30 & 3.01 & 3.00 \\
& 8 & $\mathbf{1 6 0 8 . 8 3}$ & 3.47 & 2.40 & 2.40 \\
& 10 & $\mathbf{1 7 9 9 . 1 2}$ & 1.44 & 0.83 & 0.82 \\
& 12 & $\mathbf{2 0 0 9 . 9 7}$ & 0.41 & 0.30 & 0.30 \\
\hline
\end{tabular}

existing policies are as follows: $3.56 \%$ over $P(\mathbf{s}, \mathbf{S})$ and $2.29 \%$ over $(Q, \mathbf{S})$ and $Q(\mathbf{s}, \mathbf{S})$. A peculiarity if the $Q(\mathbf{s}, \mathbf{S})$ policy strikes out immediately: Incorporation of individual trigger levels $\mathbf{s}$ does not improve the much simpler $(Q, \mathbf{S})$ policy noticeably in the case of identical items. In comparison with the $(Q, \mathbf{S})$ policy, we observe, however, that introduction of a time trigger in the $(Q, \mathbf{S}, T)$ policy provides significant savings. The savings under the proposed policy are much pronounced for a small number of items. As $N$ grows large, the difference between the performances of the policies starts diminishing; however, $P(\mathbf{s}, \mathbf{S})$ is much slower in this respect. We observe that the effect of lead time is not monotone. We should also report that in the tabulated cases the optimal system fill rates are in the neighborhood of $99 \%$.

The last issue we investigate is the impact of demand rate diversity among the items on the policy performances. In Table 8, we report a representative case of $N=4$ items with identical cost parameters of $K=150, \pi=30, \rho=0, k=$ 20 , and $h=6$ and identical lead times of $L=0.2$ and a system demand rate of $\lambda_{0}=320$. As tabulated, we consider various groupings of demand rates among the items. In the first block (instance 1), all items have equal demand rates and it may be viewed as a reference instance. The rest of the instances attempt to generate groupings of differing demand rate diversity among the items, producing "lop-sided" spread of demands. In the second and third blocks (instances 2 through 5 and 10 through 13), three items are identical and one is different. In the third block (instances 6 through 9), items are grouped into two identical pairs. In the last block (instances 14 through 18), all four items have different demand rates.

The proposed policy dominates the existing policies in this set as well. The average savings achieved through the implementation of the proposed policy in lieu of each of the existing policies are as follows: $3.76 \%$ over $P(\mathbf{s}, \mathbf{S}), 3.88 \%$ over $(Q, \mathbf{S})$, and $2.66 \%$ over $Q(\mathbf{s}, \mathbf{S})$. The average savings over the next best policy is $2.60 \%$.

For the improvement achieved through the proposed policy we make the following observations. For the first 13 instances, we see that, as diversity among item demand rates increases, the savings of the proposed policy also increases. For the last block, the opposite is true when we compare the proposed policy against $P(\mathbf{s}, \mathbf{S})$ and $Q(\mathbf{s}, \mathbf{S})$. Furthermore, for the last block, the improvement is significantly smaller

Table 8. Performance of $(Q, \mathbf{S}, T)$ policy for non-identical items-additional set, $K=150, k=20, h=6, \pi=30, \rho=0$.

\begin{tabular}{|c|c|c|c|c|c|c|c|}
\hline$\lambda_{1}$ & $\lambda_{2}$ & $\lambda_{3}$ & $\lambda_{4}$ & $A C_{(Q, \mathbf{S}, T)}$ & $\Delta_{P(\mathbf{s}, \mathbf{S})} \%$ & $\Delta_{(Q, \mathbf{S})} \%$ & $\Delta_{Q(\mathbf{s}, \mathbf{S})} \%$ \\
\hline 80 & 80 & 80 & 80 & 1191.08 & 5.11 & 3.58 & 3.58 \\
\hline 70 & 70 & 70 & 110 & 1080.65 & 4.75 & 2.30 & 2.30 \\
\hline 60 & 60 & 60 & 140 & 1049.18 & 5.12 & 2.45 & 2.45 \\
\hline 50 & 50 & 50 & 170 & 1018.61 & 6.24 & 2.78 & 2.78 \\
\hline 40 & 40 & 40 & 200 & 998.95 & 6.40 & 3.12 & 3.12 \\
\hline 70 & 70 & 90 & 90 & 1131.88 & 4.12 & 3.79 & 3.78 \\
\hline 60 & 60 & 100 & 100 & 1109.69 & 4.56 & 3.98 & 3.98 \\
\hline 50 & 50 & 110 & 110 & 1087.92 & 5.09 & 4.21 & 4.21 \\
\hline 40 & 40 & 120 & 120 & 1066.60 & 5.40 & 4.34 & 4.34 \\
\hline 70 & 83.33 & 83.33 & 83.33 & 1166.47 & 3.27 & 2.66 & 2.66 \\
\hline 60 & 86.67 & 86.67 & 86.67 & 1154.93 & 4.02 & 2.77 & 2.77 \\
\hline 50 & 90 & 90 & 90 & 1143.49 & 4.30 & 2.99 & 2.99 \\
\hline 40 & 93.33 & 93.33 & 93.33 & 1132.17 & 4.55 & 3.21 & 3.21 \\
\hline 70 & 60 & 100 & 90 & 1170.55 & 1.58 & 5.27 & 1.78 \\
\hline 70 & 50 & 110 & 90 & 1173.22 & 1.31 & 5.36 & 1.56 \\
\hline 70 & 40 & 120 & 90 & 1177.38 & 0.92 & 5.49 & 1.01 \\
\hline 70 & 30 & 130 & 90 & 1179.07 & 0.55 & 5.61 & 0.77 \\
\hline 70 & 20 & 140 & 90 & 1179.53 & 0.34 & 5.93 & 0.56 \\
\hline
\end{tabular}


Table 9. Sensitivity results with respect to $K, h, \rho, \pi, L, \lambda_{0}, N=4$, and $k=20$.

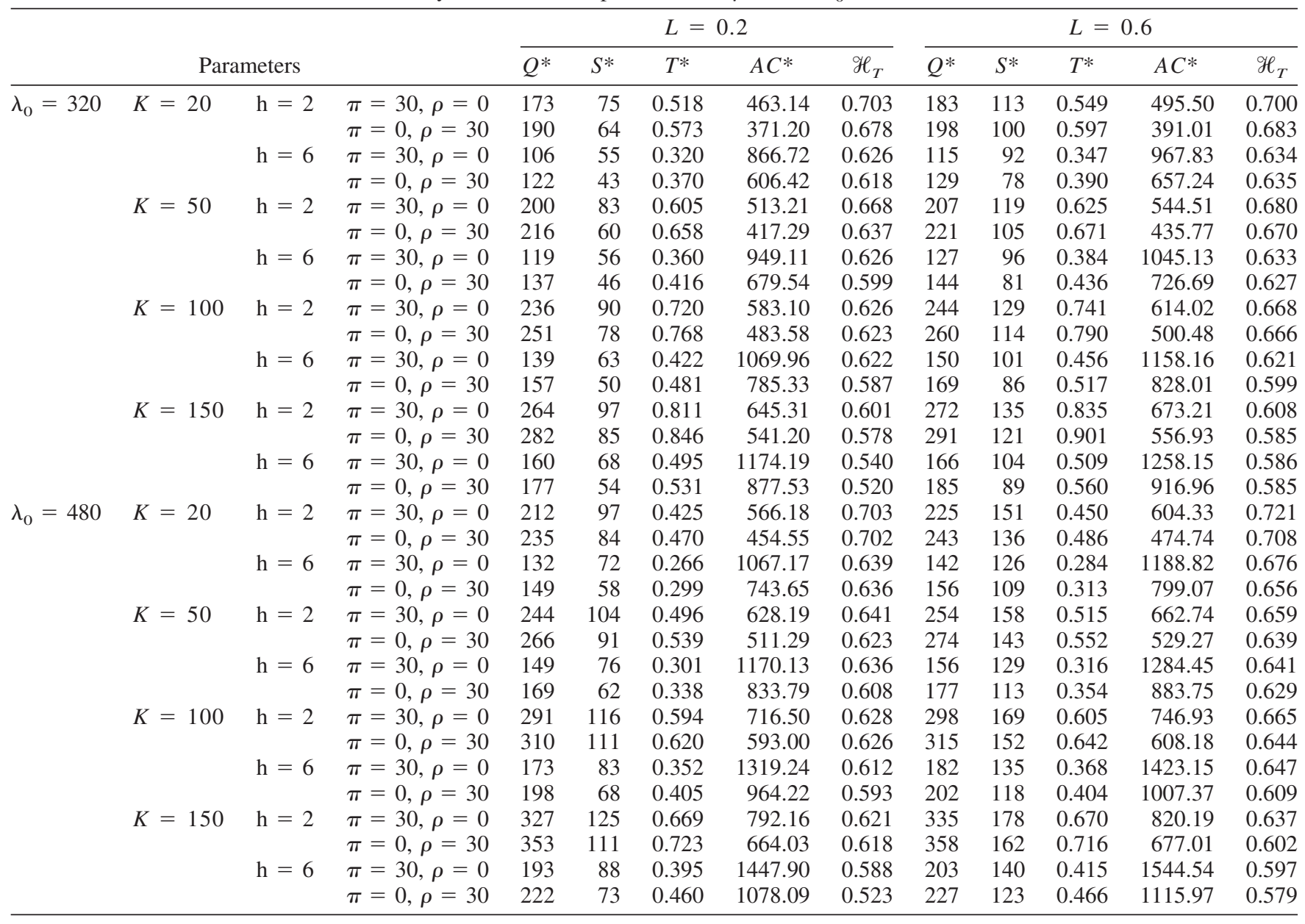

than those for other instances. We also note that as item demand rates become more dissimilar, performances of $P(\mathbf{s}$, $\mathbf{S})$ and $Q(\mathbf{s}, \mathbf{S})$ approach that of the proposed policy. Considering the next best policy, we observe an interesting change of dominance among the policies. In the first 13 instances, performances of $Q(\mathbf{s}, \mathbf{S})$ and $(Q, \mathbf{S})$ are almost identical and they both dominate $P(\mathbf{s}, \mathbf{S})$. However, in the last block (instances 14 through 18), when items have identical unit cost structures but are dissimilar greatly in their individual demand rates, we observe a shift so that $Q(\mathbf{s}, \mathbf{S})$ performs significantly better than $(Q, \mathbf{S})$. Furthermore, in the same region, $P(\mathbf{s}, \mathbf{S})$ starts to dominate $Q(\mathbf{s}, \mathbf{S})$, albeit by a small margin.

Therefore, we may conclude that the dominance of any policy is also strongly dependent on how the individual demand rates are distributed among the items. Noting that our model can represent a two-echelon, single-item, multiretailer setting with cross docking, this finding has important implications for supply chain design and management. It would be interesting to investigate the joint location- allocation-replenishment problem in a supply chain. We intend to do this in a future study.

\subsection{Sensitivity Analysis}

For the sensitivity analysis, we used an augmented experimental testbed that includes the settings considered previously in the literature. We report only a representative set in which $N=4$ and all items are assumed to be identical in their cost and demand parameters. We also consider only unit demands. Table 9 illustrates the experimental set and the results. The experimental points represent a wide range of parameters from very high service levels with low ordering and high backorder costs to lower service levels with high ordering and low backorder costs.

We focus on the behavior of the optimal policy parameter values and the expected average cost rate with respect to ordering costs, unit holding and backorder costs, lead time, and system demand rate.

Under the proposed policy, there are two reorder trigger mechanisms as discussed above. To better assess the impact 
Table 10. Performance of $(Q, \mathbf{S}, T)$ policy for identical items with different number of items and compound Poisson demand, $\alpha=320$, $L=0.2, K=150, k=20, h=6, \pi=30, \rho=0$.

\begin{tabular}{|c|c|c|c|c|c|c|}
\hline \multirow{2}{*}{$\begin{array}{c}\text { Batch dist. } \\
\quad N \\
\end{array}$} & \multicolumn{2}{|c|}{ Unit $\left(\sigma_{d}=0\right)$} & \multicolumn{2}{|c|}{$\operatorname{Geo}(p=0.05),\left(\sigma_{d}^{2}=2\right)$} & \multicolumn{2}{|c|}{$\operatorname{Geo}(p=0.2),\left(\sigma_{d}^{2}=20\right)$} \\
\hline & $A C_{(Q, \mathbf{S}, T)}$ & $\Delta_{(Q, \mathbf{S})} \%$ & $A C_{(Q, \mathbf{S}, T)}$ & $\Delta_{(Q, \mathbf{S})} \%$ & $A C_{(Q, \mathbf{S}, T)}$ & $\Delta_{(Q, \mathbf{S})} \%$ \\
\hline 2 & 1058.91 & 5.21 & 1082.34 & 6.14 & 1105.18 & 6.54 \\
\hline 4 & 1191.08 & 3.58 & 1225.67 & 4.13 & 1276.10 & 4.72 \\
\hline 6 & 1358.01 & 1.86 & 1398.98 & 2.15 & 1453.01 & 3.19 \\
\hline 8 & 1502.78 & 1.18 & 1576.14 & 1.29 & 1604.26 & 1.76 \\
\hline 10 & 1681.29 & 0.46 & 1723.09 & 1.02 & 1775.20 & 1.24 \\
\hline 12 & 1861.19 & 0.32 & 1903.21 & 0.68 & 1952.15 & 0.95 \\
\hline
\end{tabular}

of the time trigger $T$ of the proposed policy, we also report the probability $\mathscr{H}_{T}$, that a replenishment order is given by the time trigger. From Corollary 2.1.b, $\mathscr{H}_{T}$ can be calculated as

$$
\begin{aligned}
\mathscr{H}_{T}=\sum_{m=1}^{\infty} f_{Y}(m T) & =\sum_{m=1}^{\infty} \phi_{0}^{m-1}\left[P_{0}\left(Q-1, \lambda_{0} T\right)-\phi_{0}\right] \\
& =\frac{1}{1-\phi_{0}}\left[P_{0}\left(Q-1, \lambda_{0} T\right)-\phi_{0}\right] .
\end{aligned}
$$

We present the optimal policy parameters, $\left(Q^{*}, \mathbf{S}^{*}, T^{*}\right)$, the optimal cost rate function, $A C^{*}$, and $\mathcal{H}_{T}^{*}$ calculated at $\left(Q^{*}, T^{*}\right)$ in Table 9 for identical item specific ordering costs, $k_{i}=20, i=1, \ldots, 4$.

We observe that the behavior of the policy parameters with respect to system parameters is quite intuitive. We discuss them below in some detail.

The effect of increasing the common ordering cost, $K$, is to delay the order placement by increasing $Q^{*}$ and/or $T^{*}$. However, the increase in $T^{*}$ is usually more pronounced than the change in $Q^{*}$. We also note that $T^{*}$ is, in general, smaller than $Q^{*} / \lambda_{0}$, which is the average time for $Q^{*}$ demands to accumulate at the system level. Thus, $T^{*}$ acts as a pro-active trigger. But, as $K$ increases, we lose this property and $T^{*}$ becomes very close to $Q^{*} / \lambda_{0}$. The loss of the pro-activeness of the time trigger is also manifest in a decrease in $\mathscr{H}_{T}^{*}$ with increasing $K$. Increasing the common ordering cost also results in larger values of $S^{*}$ so as to avoid stockouts due to the resulting delay in the reordering decision.

As the unit holding cost increases, all optimal policy parameters decrease. That is, reordering decisions are made more frequently and inventory positions of the items are raised up to lower levels to prevent the increase in the average inventory level. At the same time, we note that $\mathcal{H}_{T}^{*}$ generally decreases with $h$. This implies that the system has a tendency to give orders as triggered by the accumulation of $Q$ demands, rather than being made at the time trigger for higher $h$.
The delivery lead time of the items and the system demand rate (or, equivalently, the individual item demand rates) have a considerable effect on the optimal policy parameters. As the lead time increases, both $Q^{*}$ and $T^{*}$ increase and the order-up-to levels get larger. However, as the system demand rate $\lambda_{0}$ increases, $Q^{*}$ and $\mathbf{S} *$ get large but $T^{*}$ gets smaller. That is, for higher demand rates, the reordering decision is triggered more frequently by the time trigger. This is to be expected because longer lead time or larger demand rates increase the risk of stock-outs during lead time, so the proactive option of the policy becomes more desirable. This is best illustrated in $\mathcal{H}_{T}^{*}$, which is increasing overall in $L$ and $\lambda_{0}$.

When unit shortage costs increase (i.e. higher service levels are desired), orders are given more frequently and the items are replenished to higher levels. $T^{*}$ decreases considerably as $\rho$ increases and as $\pi$ decreases.

As expected, the optimal cost rate, $A C^{*}$ is increasing in $K, h, \pi, L$, and $\lambda_{0}$ across the entire test bed.

\subsection{Batch Demand}

Finally, we study the impact of batch demand arrivals. For illustration, we consider the case where all items are identical in demand rate and cost parameters. The demand is assumed to follow a compound Poisson process with an overall rate $\lambda_{0} / N$ and a geometrically distributed demand size with parameter $p$ for all items. Since the rest of the policies have not been generalized for compound demand processes, we can only report the comparison between $(Q$, $\mathbf{S}, T)$ and $(Q, \mathbf{S})$, which is its special case. To make a fair comparison across different demand size parameters, we fix the average number of units demanded per time, $\lambda_{0} / p=\alpha$. We set $\alpha=320, L=0.2, K=150, k=20, h=6, \pi=$ $30, \rho=0$. We vary $p \in\{0.2,0.5\}$ and $N \in\{2,4,6$, $8,10,12\}$. We use $\sigma_{d}^{2}$ to denote the variance of demand size. We present the performance of $(Q, \mathbf{S}, T)$ policy in the presence of batch demands in Table 10 . We observe the intuitive finding that as the variance of the demand size increases, the savings due to the introduction of a time trigger also increase. As in the unit demand case, the sav- 
ings decrease with the number of items, $N$, but at a slightly slower rate. In unreported experiments, we also observed that the behavior of the cost rate with batch demands with respect to the cost parameters and the demand rates is similar to that with unit demands, as expected.

\section{CONCLUDING REMARKS}

In this study, we have proposed a new ordering policy for stochastic joint replenishment problem. The proposed policy bases the ordering decisions not only on the total demands that have accumulated in the system but also on the time that has elapsed since the last decision epoch. Under this time-based joint ordering policy, we developed expressions for the operating characteristics of the inventory system and constructed the expected cost rate function for unit and compound Poisson demand processes.

An extensive numerical study has been conducted to study the sensitivity of the policy to various system parameters and to assess the performance of the proposed policy over existing policies. The numerical experiments indicate that there is no clear demarcation of operating environments for the dominance of proposed policies in the literature and that the dominance of the proposed policy is not monotone over the experiments. However, similarity of items in their cost structure appears to be most critical factor in the dominance of the proposed policy. The diversity of the individual demand rates is also an important factor. We have found that the proposed policy provides significant savings over the existing policies for items similar in their cost structures and individual demand rates. This finding, we believe, may have important implications for supply chain design.

\section{APPENDIX}

Proof of Lemma 2.1: Let $N(t)$ be the counting process of system demands in $(0, t]$ where $t=0$ is taken as the beginning of a replenishment cycle.

First, suppose $y=m T$ for $m \geq 1$ and $0<q<Q$. This case corresponds to a replenishment cycle depicted in Figure 1a.

$$
\begin{array}{r}
P\left(Y=m T, Q_{0}=q\right)=P(N((m-1) T)=0, N(m T)-N((m-1) T) \\
=q)=P(N(T)=0)^{m-1} P(N(T)=q)=\phi_{0}^{m-1} p_{0}\left(q, \lambda_{0} T\right) \\
\quad m \geq 1,0<q<Q
\end{array}
$$

Now, suppose $(m-1) T<y<m T$ for $m \geq 1$ and $q=Q$. This case corresponds to a replenishment cycle depicted in Figure 1b. An order of size $Q_{0}=Q$ is triggered in time interval $[y, y+\delta y)$ if the following events occur: $N((m-1) T)=0, N(y)-N(y-(m-1) T)=Q-1$, and $N(y+\delta y)-N(y)=1$. Then, we have

$$
\begin{array}{r}
P\left(Y \in(y, y+\delta y], Q_{0}=Q\right)=P(N((m-1) T)=0, N(y)-N(y-(m \\
-1) T)=Q-1, N(y+\delta y)-N(y)=1)=\phi_{0}^{m-1} p_{0}\left(Q-1, \lambda_{0}(y-(m\right. \\
-1) T)) \lambda_{0}[\delta y+o(\delta y)] .
\end{array}
$$

Result follows by dividing both sides by $\delta y$ and taking the limit as $\delta y \rightarrow$ 0 .

Proof of Proposition 2.1: For $t$ and $1 \leq i \leq N$, let $\xi_{i}(t)=\left\{t, N_{i}, N_{0}\right.$, $Z$ \} be defined as the three-dimensional stochastic process, where $Z$ denotes the time elapsed between $t$ and the last decision epoch, $N_{i}$ and $N_{0}$ denote the number of demands for item $i$ and the number of demands for all items other than $i$, respectively, that have arrived over $Z$ time units.

A particular state that $\xi_{i}(t)$ will visit at time $t$ will be denoted by $\left\{n_{i}, n_{0}\right.$, $z$. Then, $g_{i}\left(t, n_{i}, n_{0}, z\right)$ denotes the corresponding probability density function of $\xi_{i}(t)$ being in state $\left\{n_{i}, n_{0}, z\right\}$ at time $t$. We first derive the partial differential equations that $g_{i}\left(t, n_{i}, n_{0}, z\right)$ satisfies and use them to obtain the partial differential equations for the steady-state distribution, $g_{i}\left(n_{i}, n_{0}, z\right)$. We derive the equations for four different cases and state the boundary condition.

$$
\begin{aligned}
& \text { Case } 1: n_{0}=0, n_{i}=0,0<z<T . \\
& \qquad g_{i}\left(t+\delta_{t}, 0,0, z+\delta t\right)=g_{i}(t, 0,0, z)\left(1-\lambda_{0} \delta t\right)+o(\delta t),
\end{aligned}
$$

where $o(\delta t) / \delta t \rightarrow 0$ as $\delta t \rightarrow 0$. This follows because the state of item $i$ will be $(0,0, z+\delta t)$ at time $t+\delta t$ if it is in state $(0,0, z)$ at time $t$ and no demands arrive for any of the items during the interval $[t, t+\delta t)$ which has probability $1-\lambda_{0} \delta t+o(\delta t)$. For sufficiently small $\delta t, z+\delta t<T$ should also hold so that a review is not carried out.

Subtracting the term $g_{i}(t, 0,0, z+\delta t)$ from both sides and dividing both sides by $\delta t$ and letting $\delta t \rightarrow 0$ gives

$$
\frac{\partial g_{i}(t, 0,0, z)}{\partial t}=-\frac{\partial g_{i}(t, 0,0, z)}{\partial z}+\lambda_{0} g_{i}(t, 0,0, z)
$$

Taking the limit as $t \rightarrow \infty$ results in

$$
\frac{\partial g_{i}(0,0, z)}{\partial z}=-\lambda_{0} g_{i}(0,0, z) \quad 0<z<T .
$$

Case 2. $n_{0}=0,0<n_{i}<Q$, and $0<z<T$,

$$
\begin{aligned}
g_{i}\left(t+\delta_{t}, n_{i}, 0, z+\delta t\right)=g_{i}\left(t, n_{i}, 0, z\right)\left(1-\lambda_{0} \delta t\right) & \\
& +g_{i}\left(t, n_{i}-1,0, z\right) \lambda_{i} \delta t+o(\delta t) .
\end{aligned}
$$

The state of item $i$ will be $\left(n_{i}, 0, z+\delta t\right)$ at time $t+\delta t$ either if at time $t$ the state is $\left(n_{i}, 0, z\right)$ and no demands arrive in $[t, t+\delta t)$; or the state at time $t$ is $\left(n_{i}-1,0, z\right)$ and a demand arrives for item $i$ during the interval $[t, t+\delta t)$ with probability $\lambda_{i} \delta t+o(\delta t)$. Subtracting the term $g_{i}\left(t, n_{i}, 0, z+\delta t\right)$ from both sides and dividing both sides by $\delta t$ and letting $\delta t \rightarrow 0$ results in

$$
\frac{\partial g_{i}\left(t, n_{i}, 0, z\right)}{\partial t}=-\frac{\partial g_{i}\left(t, n_{i}, 0, z\right)}{\partial z}-\lambda_{0} g_{i}\left(t, n_{i}, 0, z\right)+\lambda_{i} g_{i}\left(t, n_{i}-1,0, z\right) .
$$

Then, letting $t \rightarrow \infty$,

$$
\frac{\partial g_{i}\left(n_{i}, 0, z\right)}{\partial z}=-\lambda_{0} g_{i}\left(n_{i}, 0, z\right)+\lambda_{i} g_{i}\left(n_{i}-1,0, z\right) \quad 0<n_{i}<Q, z<T .
$$

Case 3: $0<n_{0}<Q, n_{i}=0$, and $0<z<T$,

$g_{i}\left(t+\delta_{t}, 0, n_{0}, z+\delta t\right)=g_{i}\left(t, 0, n_{0}, z\right)\left(1-\lambda_{0} \delta t\right)+g_{i}\left(t, 0, n_{o}-1, z\right)$

$$
\times\left(\lambda_{0}-\lambda_{i}\right) \delta t+o(\delta t) \text {. }
$$


This case is very similar to Case 2 and no further details will be given except the following partial differential equation for the steady-state probability density function:

$$
\begin{aligned}
\frac{\partial g_{i}\left(0, n_{0}, z\right)}{\partial z}=-\lambda_{0} g_{i}\left(0, n_{0}, z\right)+\left(\lambda_{0}-\lambda_{i}\right) g_{i}\left(0, n_{0}-1, z\right) & \\
& 0<n_{0}<Q, z<T
\end{aligned}
$$

Case 4: $0<n_{0}<Q, 0<n_{i}<Q, 0<n_{i}+n_{0}<Q$, and $0<z<$ $T$,

$$
\begin{aligned}
& g_{i}\left(t+\delta_{t}, n_{i}, n_{0}, z+\delta t\right)=g_{i}\left(t, n_{i}, n_{0}, z\right)\left(1-\lambda_{0} \delta t\right)+g_{i}\left(t, n_{i}\right. \\
&\left.-1, n_{0}, z\right) \lambda_{i} \delta t+g_{i}\left(t, n_{i}-1, n_{0}, z\right)\left(\lambda_{0}-\lambda_{i}\right) \delta t+o(\delta t) .
\end{aligned}
$$

This follows because the state at time $t+\delta t$ will be $\left(n_{i}, n_{0}, z+\delta t\right)$ only if one of the following three events occur: the state at time $t$ is $\left(n_{i}, n_{0}, z\right)$ and no demands arrive at the system in $[t, t+\delta t)$; the state at time $t$ is $\left(n_{i}-1, n_{0}, z\right)$ and a demand arrives for item $i$ in $[t, t+\delta t)$; the state is $\left(n_{i}, n_{0}-1, z\right)$ at time $t$ and a demand arrives for an item other than $i$ in $[t, t+\delta t)$.

Subtracting the term $g_{i}\left(t, n_{i}, n_{0}, z+\delta t\right)$ from both sides, dividing by $\delta t$, and letting $\delta t \rightarrow 0$ results in

$$
\begin{aligned}
& \frac{\partial g_{i}\left(t, n_{i}, n_{0}, z\right)}{\partial t}=-\frac{\partial g_{i}\left(t, n_{i}, n_{0}, z\right)}{\partial z}- \lambda_{0} g_{i}\left(t, n_{i}, n_{0}, z\right)+\lambda_{i} g_{i}\left(t, n_{i}\right. \\
&\left.-1, n_{0}, z\right)+\left(\lambda_{0}-\lambda_{i}\right) g_{i}\left(t, n_{i}, n_{0}-1, z\right) .
\end{aligned}
$$

Finally, $t \rightarrow \infty$, we have

$$
\begin{gathered}
\frac{\partial g_{i}\left(n_{i}, n_{0}, z\right)}{\partial z}=-\lambda_{0} g_{i}\left(n_{i}, n_{0}, z\right)+\lambda_{i} g_{i}\left(n_{i}-1, n_{0}, z\right)+\left(\lambda_{0}-\lambda_{i}\right) g_{i}\left(n_{i}, n_{0}\right. \\
-1, z) \quad 0<n_{i}<Q, 0<n_{0}<Q, 0<n_{i}+n_{0}<Q, 0<z<T
\end{gathered}
$$

The boundary condition to the system of partial differential equations described above is as follows:

$g_{i}(0,0,0)=\sum_{n_{i}=0}^{Q-1} \int_{z=0}^{T} \lambda_{0} g_{i}\left(n_{i}, Q-1-n_{i}, z\right) d z$

$$
+\sum_{n_{i}=0}^{Q-1} \sum_{n_{0}=0}^{Q-1-n_{i}} \lim _{z \rightarrow T} g_{i}\left(n_{i}, n_{0}, z\right)
$$

We now verify that the proposed solution (1) satisfies Eqs. (15)-(18) given above.

Case 1: $n_{0}=n_{i}=0,0<z<T$

$$
\begin{aligned}
g_{i}(0,0, z) & =C_{0} e^{-\lambda_{0} z} \\
\frac{\partial g_{i}(0,0, z)}{\partial z} & =-C_{0} \lambda_{0} e^{-\lambda_{0} z}=-\lambda_{0} g_{i}(0,0, z)
\end{aligned}
$$

Case 2: $n_{0}=0,0<n_{i}<Q, 0<z<T$

$$
\begin{aligned}
g_{i}\left(n_{i}, 0, z\right) & =C_{0} e^{-\lambda_{0} z} \frac{\left(\lambda_{i} z\right)^{n_{i}}}{n_{i} !} \\
\frac{\partial g_{i}\left(n_{i}, 0, z\right)}{\partial z} & =-\lambda_{0} C_{0} e^{-\lambda_{0} z} \frac{\left(\lambda_{i} z\right)^{n_{i}}}{n_{i} !}+C_{0} e^{-\lambda_{0} z} \lambda_{i} \frac{\left(\lambda_{i} z\right)^{n_{i}-1}}{\left(n_{i}-1\right) !} \\
& =-\lambda_{0} g_{i}\left(n_{i}, 0, z\right)+\lambda_{i} g_{i}\left(n_{i}-1,0, z\right)
\end{aligned}
$$

Case 3: $0<n_{0}<Q, n_{i}=0,0<z<T$

$$
\begin{aligned}
g_{i}\left(0, n_{0}, z\right) & =C_{0} e^{-\lambda_{0} z} \frac{\left(\left(\lambda_{0}-\lambda_{i}\right) z\right)^{n_{0}}}{n_{0} !} \\
\frac{\partial g_{i}\left(0, n_{0}, z\right)}{\partial z} & =-\lambda_{0} C_{0} e^{-\lambda 0 z} \frac{\left(\left(\lambda_{0}-\lambda_{i}\right) z\right)^{n_{0}}}{n_{0} !} \\
+C_{0} e^{-\lambda_{0 z}}\left(\lambda_{0}-\lambda_{i}\right) \frac{\left(\left(\lambda_{0}-\lambda_{i}\right) z\right)^{n_{0}-1}}{\left(n_{0}-1\right) !} & \\
& =-\lambda_{0} g_{i}\left(0, n_{0}, z\right)+\left(\lambda_{0}-\lambda_{i}\right) g_{i}\left(0, n_{0}-1, z\right)
\end{aligned}
$$

Case 4: $0<n_{0}<Q, 0<n_{i}<Q, 0<z<T$

$$
g_{i}\left(n_{i}, n_{0}, z\right)=C_{0} e^{-\lambda_{0} z} \frac{\left(\lambda_{i} z\right)^{n_{i}}}{n_{i} !} \frac{\left(\left(\lambda_{0}-\lambda_{i}\right) z\right)^{n_{0}}}{n_{0} !}
$$

$$
\begin{aligned}
\frac{\partial g_{i}\left(n_{i}, n_{0}, z\right)}{\partial z} & =-\lambda_{0} C_{0} e^{-\lambda_{0} z} \frac{\left(\lambda_{i} z\right)^{n_{i}}}{n_{i} !} \frac{\left(\left(\lambda_{0}-\lambda_{i}\right) z\right)^{n_{0}}}{n_{0} !} \\
& +\lambda_{i} C_{0} e^{-\lambda_{0} z} \frac{\left(\lambda_{i} z\right)^{n_{i}-1}}{\left(n_{i}-1\right) !}+\left(\lambda_{0}-\lambda_{i}\right) C_{0} e^{-\lambda_{0} z} \frac{\left(\left(\lambda_{0}-\lambda_{i}\right) z\right)^{n_{0}-1}}{\left(n_{0}-1\right) !} \\
& =-\lambda_{0} g_{i}\left(n_{i}, n_{0}, z\right)+\lambda_{i} g_{i}\left(n_{i}-1, n_{0}, z\right) \\
& +\left(\lambda_{0}-\lambda_{i}\right) g_{i}\left(n_{i}, n_{0}-1, z\right)
\end{aligned}
$$

Thus, the steady-state probability density function has the structure given in Eq. (1). Moreover, for $g_{i}\left(n_{i}, n_{0}, z\right)$ to be a probability density function,

$$
\int_{z=0}^{T}\left[\sum_{n_{i}=0}^{Q-1} \sum_{n_{0}=0}^{Q-1-n_{i}} g_{i}\left(n_{i}, n_{0}, z\right)\right] d z=1 .
$$

Therefore,

$$
\begin{aligned}
\int_{z=0}^{T}\left[\sum_{n_{i}=0}^{Q-1} C_{0} p_{0}\left(n_{i}, \lambda_{i} z\right) P_{0}\left(Q-1-n_{i},\left(\lambda_{0}-\lambda_{i}\right) z\right)\right] d z & =\int_{z=0}^{T} C_{0} P_{0}(Q \\
& \left.-1, \lambda_{0} z\right) d z=1 .
\end{aligned}
$$

Hence,

$$
C_{0}=\left[\int_{z=0}^{T} P_{0}\left(Q-1, \lambda_{0} z\right) d z\right]^{-1} .
$$




\section{Derivation of Eq. 2}

Using Corollary 2.1 we can write

$$
\begin{array}{r}
E[Y]=\sum_{m=1}^{\infty} m T \phi_{0}^{m-1}\left[P_{0}\left(Q-1, \lambda_{0} T\right)-\phi_{0}\right]+\sum_{m=1}^{\infty} \int_{y=(m-1) T}^{m T} \phi_{0}^{m-1} y f\left(y-(m-1) T, Q, \lambda_{0}\right) d y=\frac{T\left[P_{0}\left(Q-1, \lambda_{0} T\right)-\phi_{0}\right]}{\left(1-\phi_{0}\right)^{2}}+\sum_{m=1}^{\infty} \phi_{0}^{m-1} \int_{t=0}^{T}(t+(m \\
-1) T) f\left(t, Q, \lambda_{0}\right) d t=\frac{T\left[P_{0}\left(Q-1, \lambda_{0} T\right)-\phi_{0}\right]}{\left(1-\phi_{0}\right)^{2}}+\frac{1}{1-\phi_{0}} \int_{t=0}^{T} t f\left(t, Q, \lambda_{0}\right) d t+\sum_{m=1}^{\infty}(m-1) \phi_{0}^{m-1} T F\left(T, Q, \lambda_{0}\right)=\frac{T P_{0}\left(Q-1, \lambda_{0} T\right)-T \phi_{0}}{\left(1-\phi_{0}\right)^{2}} \\
+\frac{Q}{\lambda_{0}\left(1-\phi_{0}\right)} \int_{t=0}^{T} f\left(t, Q+1, \lambda_{0}\right) d t+\sum_{m=1}^{\infty} m \phi_{0}^{m-1} T F\left(T, Q, \lambda_{0}\right)-\sum_{m=1}^{\infty} \phi_{0}^{m-1} T F\left(T, Q, \lambda_{0}\right)=\frac{T P_{0}\left(Q-1, \lambda_{0} T\right)-T \phi_{0}}{\left(1-\phi_{0}\right)^{2}}+\frac{Q}{\lambda_{0}\left(1-\phi_{0}\right)} F\left(T, Q+1, \lambda_{0}\right) \\
+\frac{T}{\left(1-\phi_{0}\right)^{2}} F\left(T, Q, \lambda_{0}\right)-\frac{T}{1-\phi_{0}} F\left(T, Q, \lambda_{0}\right)=\frac{T P_{0}\left(Q-1, \lambda_{0} T\right)}{1-\phi_{0}}+\frac{Q \bar{P}_{0}\left(Q, \lambda_{0} T\right)}{\lambda_{0}\left(1-\phi_{0}\right)} .
\end{array}
$$

\section{Search Algorithm}

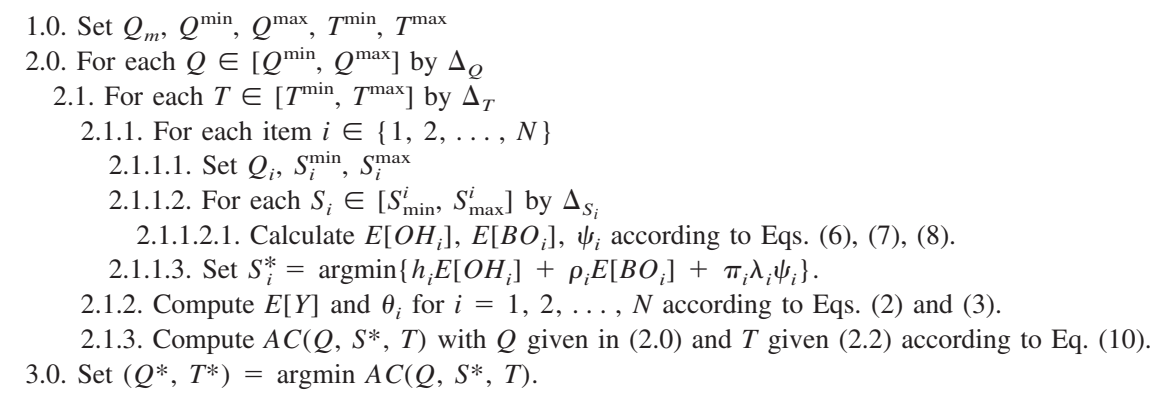

\section{REFERENCES}

[1] Y. Aksoy and S. Erenguc, Multi-item models with coordinated replenishments: A survey, Int J Prod Manage 8 (1988), 63-73.

[2] D.R. Atkins and P.O. Iyogun, A lower bound for a class of inventory/production problems, Oper Res Lett 6 (1987), 63-67.

[3] D.R. Atkins and P.O. Iyogun, Periodic versus can-order policies for coordinated multi-item inventory systems, Manage Sci 34 (1988), 791-796.

[4] J.L. Balintfy, On a basic class on inventory problems, Manage Sci 10 (1964), 287-297.

[5] G. Cachon, Managing a retailer's shelf space, inventory and transportation, Manufact Serv Oper Manage 3 (2001), 211-229.

[6] P.D. Cousin, Supply base rationalisation: myth or reality? Eur J Purchas Supply Manage 5 (1999), 143-155.

[7] D.R. Cox, "The analysis of non-markovian stochastic processes by the inclusion of supplementary variables," Proceedings of the Cambridge Philosophical Society, 1955, Vol. 51, pp. $433-441$.

[8] F. Erhun and S. Tayur, Enterprise-wide optimization of total landed cost at a grocery retailer, Oper Res 51 (2003), 343-353.

[9] A. Federgruen, H. Greoenevelt, and H. Tijms, Coordinated replenishments in a multi-item inventory system with com- pound poisson demands and constant lead times, Manage Sci 30 (1984), 344-357.

[10] A. Federgruen and Y.S. Zheng, The joint replenishment problem with general joint cost structures, Oper Res 40 (1992), 384-403.

[11] M.L. Fisher, J.H. Hammond, W.R. Obermeyer, and A. Raman, Making supply meet demand in an uncertain world, Harvard Bus Rev 72 (1994), 83-93.

[12] S.H. Goyal and A.T. Satir, Joint replenishment inventory control, Eur J Oper Res 38 (1989), 2-13.

[13] G. Hadley and T.M. Whitin, Analysis of Inventory Systems. Prentice Hall, NJ, 1963.

[14] T.M. Harland, Supply chain management: relationships, chains and networks, Br J Manage 7 (1996), 63-80.

[15] E. Ignall, Optimal continuous review policies for two product inventory systems with joint set-up costs, Manage Sci 15 (1969), 278-183.

[16] S.G. Johansen and P. Melchiors, Can-order policy for the periodic review joint replenishment problem, J Oper Res Soc 54 (2003), 283-290.

[17] K. Katircioglu, Essays in Inventory Control, $\mathrm{PhD}$ thesis, University of British Columbia, 1996.

[18] P. Melchiors, Calculating can-order policies for the joint replenishment problem by the compensation approach, Eur J Oper Res 141 (2002), 587-595. 
[19] K. Moinzadeh, An improved ordering policy for continuous review inventory system with arbitrary inter-demand time distributions, IIE Trans 33 (2001), 111-118.

[20] C. Nielsen and C. Larsen, An analytical study of the $q(s, s)$ policy applied to the joint replenishment problem, Eur J Oper Res 163 (2005), 721-732.

[21] P. Pantumsinchai, A comparison of three joint ordering policies, Decision Sci 23 (1992), 111-127.

[22] B. Renberg and R. Planche, Un modéle pour la gestion simultanée des $\mathrm{n}$ articles d'un stock, Rev Franc Inform Rech Opér 6 (1967), 47-59.

[23] C. Schmidt and S. Nahmias, $(s-1, s)$ policies for perishable inventory, Manage Sci 31 (1985), 719-728.

[24] H. Schultz and S.G. Johansen, Can-order policies for coordinated inventory replenishment with erlang distributed times between ordering, Eur J Oper Res 113 (1999), 30-41.

[25] E.A. Silver, Some characteristics of a special joint-order inventory model, Oper Res 13 (1965), 319-322.

[26] E.A. Silver, Three ways of obtaining the average cost expressions in a problem related to joint replenishment inventory control, Nav Res Logist Q 20 (1973), $241-254$.
[27] E.A. Silver, A control system for coordinated inventory replenishment, Int J Prod Res 12 (1974), 647-671.

[28] E.A. Silver, Establishing reorder points in the $(s, c, s)$ coordinated control system under compound poisson demand, Int J Prod Res 19 (1981), 743-750.

[29] S. Stidham, Stochastic clearing systems, Stoch Proc Appl 2 (1974), 85-113.

[30] E. Tekin, Ü. Gürler, and E. Berk, Age-based vs. stock level control policies for a perishable inventory system, Eur J Oper Res 134 (2001), 309-329.

[31] R.M. Thompstone and E.A. Silver, A coordinated inventory control system under compound poisson demand and zero lead time, Int J Prod Res 13 (1975), 581-602.

[32] M.J.G. Van Eijs, On the determination of the control parameters of the optimal can-order policy, Zeits Oper Res 44 (1994), 185-191.

[33] S. Viswanathan, Periodic review $(s, s)$ policies for joint replenishment inventory systems, Manage Sci 43 (1997), 1447-1454.

[34] Y.S. Zheng, Optimal control policy for stochastic inventory system with markovian discount opportunities, Oper Res 42 (1994), 721-738. 